\title{
Incorporating Dendrite Growth into Continuum Models of Electrolytes: Insights from NMR Measurements and Inverse Modelling
}

\author{
Athinthra K. Sethurajan ${ }^{1}$, Jamie M. Foster ${ }^{2}$, Giles Richardson ${ }^{3}$, \\ Sergey A. Krachkovskiy ${ }^{4}$, J. David Bazak ${ }^{4}$, \\ Gillian R. Goward ${ }^{4}$, Bartosz Protas ${ }^{*, 5}$ \\ ${ }^{1}$ School of Computational Science and Engineering, McMaster University, Canada. \\ 2 School of Mathematics and Physics, University of Portsmouth, UK. \\ ${ }^{3}$ Department of Mathematical Sciences, University of Southampton, UK. \\ ${ }^{4}$ Department of Chemistry and Chemical Biology, McMaster University, Canada. \\ ${ }^{5}$ Department of Mathematics and Statistics, McMaster University, Canada.
}

May 22, 2019

\begin{abstract}
In this work we develop a combined experimental and inverse continuum modelling approach to the problem of determining properties of a lithium electrolyte from NMR measurements of ion concentration in a test cell. The experimental set-up consists of an enclosed, lithium-electrolyte-filled tube with lithium electrodes at either end. A constant current is passed between these electrodes and the resulting evolution of the spatial distribution of the lithium ions is monitored using NMR imaging techniques. Using the recently developed tools of inverse modelling, in combination with the concentration measurements acquired with NMR imaging, it is shown that the standard PlanckNernst electrolyte model results in predictions of negative transference numbers. The observation of growing lithium dendrites on the cathode suggests the cause for these unphysical predictions and motivates the formulation of a generalized Planck-Nernst model that explicitly accounts for the presence of these growing lithium-metal dendrites. In this approach, lithium depletion in a dendritic region adjacent to the cathode is modelled by adding a suitably-chosen spatially distributed sink term. It is demonstrated that a model in which lithium is lost from the electrolyte uniformly throughout the dendritic region provides predictions of electrolyte data consistent with the literature and thereby remedies the shortcoming of the standard Planck-Nernst model. In addition, a state-of-the-art Bayesian technique is used to quantify the uncertainty of the inferred material properties.
\end{abstract}

\section{Introduction}

Lithium-ion batteries deserve significant credit for the rising popularity of portable electronic devices, power tools and electric vehicles. Methods of mathematical modeling have played an ever expanding role in research and development efforts resulting in consistent improvements in the performance and capacity of such batteries. One such specific area is the design of modern battery management systems $[9,25]$ for hybrid and electric vehicles. The key objective of mathematical modelling in this context is to quantify the transport processes involving charged species taking place in electrochemical systems

${ }^{*}$ Corresponding Author, Email: bprotas@mcmaster.ca 
at various time- and length-scales. For example, in Li-ion batteries, electrolytes enable the transport of $\mathrm{Li}$ ions between the electrodes and the knowledge of their different material properties affecting the transport processes under different operating conditions is essential for the design and calibration of predictive battery models [51]. The key material properties characterizing an electrolyte are its conductivity as well as the diffusivity and the transference number of its ions. Even for commonly used electrolytes obtaining these material properties from first principles is extremely challenging and they usually have to be measured experimentally using specialized techniques [22], a task further complicated by the fact that these material properties tend to depend strongly on the concentration of the electrolyte. This problem was studied in [47] where the concentration gradient of an Li salt was calculated based on the cell overpolarization with respect to the applied current. On the other hand, the NMR technique provides direct information about the distribution of the species in the sample. Following the seminal work of Klett et al. [20], we have developed an approach in which constant or concentration-dependent material properties are inferred indirectly by combining time- and space-resolved measurements of the concentration (obtained, for example, using NMR) with a mathematical model of the problem via a procedure referred to as "inverse modelling" [40]. In addition to providing mathematically optimal estimates of the material properties, this approach can also serve as a model-validation tool. More specifically, when the reconstructed material properties for which the model predictions best fit the experimental data are not physically consistent, this signals the loss of validity of the model [36].

In the present investigation we use the inverse-modelling approach to probe the validity of a family of mathematical models for the transport of charged species in electrolytes at moderate concentrations when certain physical effects not accounted for by the standard Planck-Nernst theory are present [31]. Once such a physically-consistent model is identified, it is used to infer optimal estimates of the diffusivity and the transference numbers (these estimates are optimal in a mathematically precise sense, because they minimize suitably defined prediction errors). Our focus here is on a canonical electrochemical experiment with a constant current applied across the electrodes [14, 35] which is often described in terms of the Planck-Nernst theory based on the following assumptions

A1: isothermal conditions;

A2: the driving force for mass transport of a species is the gradient of its chemical potential;

A3: lack of thermodynamic ideality, i.e., activity coefficients may not be identically equal to one and the effect of the solution viscosity has an a priori undetermined dependence on the salt concentration;

A4: ion transport occurs only in the axial direction and transport in the radial direction of the cell is negligible;

A5: the electrolyte solution is homogeneous at the beginning of the experiment;

A6: the system satisfies local electrical neutrality at every location in the bulk, which implies that $c_{+}=c_{-}=c$, where $c, c_{+}$and $c_{-}$are the salt, anion and lithium-ion concentrations, outside the narrow double layers near the electrodes;

A7: mass transport occurs only by diffusion and migration under the applied electric field (i.e., convective transport by fluid motion is neglected);

A8: the lithium-ion flux at the two electrodes $(x=0$ and $x=L)$ is proportional to the applied electric current and results in planar lithium deposition and stripping, respectively [31, 34].

For electrolyte in a cell of length $L$, cf. figure 1(a), cross sectional area $A$ and with constant current $I$ applied between $x=0$ and $x=L$, we can then eliminate the variables corresponding to the ionic and diffusive conductivity and write the Plank-Nernst model for the transport of the charged species in the 
following standard form [31]

$$
\begin{aligned}
\frac{\partial c}{\partial t} & =\frac{\partial}{\partial x}\left[D \frac{\partial c}{\partial x}+\frac{\left(1-t^{+}\right) I}{F A}\right] & & \text { in }(0, L) \times(0, T], \\
\left.\frac{\partial c}{\partial x}\right|_{x=0, L} & =-\frac{\left(1-t^{+}\right) I}{D F A} & & \text { in }(0, T], \\
\left.c\right|_{t=0} & =c^{\text {init }} & & \text { in }(0, L),
\end{aligned}
$$

where $D$ is the diffusivity, $t^{+}$is the transference number, $T$ is total duration of the experiment, $F$ is Faraday's constant and $c^{\text {init }}$ is the (uniform) concentration of the electrolyte at the beginning of the experiment. While constant values of $D$ and $t^{+}$correspond to the dilute limit (small concentrations), concentration-dependent material properties $D(c)$ and $t^{+}(c)$ are used to effectively account for departures from the ideal behavior. It is important to note that while transport processes in concentrated solutions are normally described based on the Onsanger-Stefan-Maxwell (OSM) constitutive relations [29], this description is in fact formally equivalent to the Planck-Nernst model with concentrationdependent material properties $D=D(c)$ and $t^{+}=t^{+}(c)$. The transference number as defined by the OSM constitutive relation is a function of the Stefan-Maxwell diffusion coefficients, however, in our model we do not make such an assumption. In this study we focus on reconstructing the diffusivity and the transference number, and do not consider the ionic conductivity and the diffusional conductivity which do not explicitly appear in system (1), but are required to fully characterize the transport properties of binary electrolytes. In an earlier study [40] we were able to infer consistent material properties using inverse modelling based on this model and an experiment featuring a rather narrow range of concentrations between $900 \mathrm{mols} / \mathrm{m}^{3}$ to $1100 \mathrm{mols} / \mathrm{m}^{3}$, demonstrating that system (1) provides an accurate description of transport phenomena in this regime. On the other hand, application of system (1) to model an experiment with a larger range of concentrations resulting from a higher applied current $I$ leads to physically inconsistent negative transference numbers obtained via inverse modelling (these results are reviewed in more detail further below). The fact that such unphysical material properties are needed by the Planck-Nernst model to accurately reproduce experimental measurements demonstrates that system (1) does not adequately describe the experiment. The reason for this is that the Planck-Nernst model (1) does not account for some physical effects affecting transport in experiments conducted at higher concentrations. One such phenomenon is the formation of ion pairs and higherorder ion clusters, however, as was shown systematically in [36], this effect does not in fact influence the transference numbers inferred from the measured concentration profiles via inverse modelling. As regards the possible influence of advection effects [24], where there is a motion induced in the liquid owing to the intrusion (recession) of plating (stripping) electrode, based on the current of $75 \mu \mathrm{A}$ and a tube diameter of $5 \mathrm{~mm}$, we estimate that if the electrode interface were to remain planar (consistent with assumption A4) and metallic, then the velocity of the interface, $\dot{s}$, would be about $7.29 \AA / \mathrm{sec}$. By then comparing the sizes of the fluxes driven by diffusion $(D c / L)$, migration $\left(\left(1-t^{+}\right) I /(F A)\right)$ and advection $(\dot{s} c)$, we find that advective effects are insignificant (driving fluxes around one hundred times smaller) compared to diffusion or migration.

In the present study we focus on another effect, namely, deposition of lithium from the electrolyte in the form of dendritic structures in a region adjacent to the cathode at $x=L[32,48]$. This phenomenon leads to the formation of a porous matrix influencing lithium transport in the affected region, cf. Figure 1 , and has been the subject of numerous investigations surveyed in the recent monograph [50] (similar investigations have also focused on electrodes made from different metals [5]). Some studies reported that when a single dendrite grows from the substrate, deposition occurs on its tip due to the concentration of the electric field $[2,3,13,28]$. However, because of their plastic deformation, the dendrites detach from the substrate, or from one another, and then deposition transitions to base-controlled growth $[15,19]$. On the other hand, clear experimental evidence of $\mathrm{Li}$ deposition occurring in $\mathrm{LiPF}_{6}-\mathrm{EC} / \mathrm{DMC}$ and resulting in "mossy" dendrite growth was provided by Steiger et al. [43] where the authors documented mechanisms for lithium deposition away from the base and dendrite tips. Two different types of Li deposition, namely, mossy and dendritic, were investigated experimentally in [4] where it was shown 
that the form of deposition depends on the current density, time and capacity of the cell. In [49] the morphology of the dendrite growth was found to be correlated with voltage profiles of the cell, whereas in [27] it was observed that electrolytes trapped inside the dendritic region cause polarization and result in complex growth patterns of the dendrites. Finally, possibilities for an accurate experimental characterization of such dendritic structures using MRI were recently discussed in [18]. Given these different observations, in our investigation we consider different ways of macroscopically accounting for the effect of lithium deposition in a modified Planck-Nernst model and demonstrate, using inverse modelling, that one of these proposed approaches does indeed lead to physically consistent reconstructions of the material properties and to accurate predictions of the evolution of the concentration profiles in time.

In this study we consider a standard electrolyte, lithium hexa-fluro phosphate $\mathrm{LiPF}_{6}$, dissolved in 1:1 mixture (by volume) of Ethylene Carbonate (EC) and Di-Methyl Carbonate (DMC), which is often used in batteries with additives. Determination of the material properties, diffusivity and the transference number, of this electrolyte has been the subject of many investigations $[1,17,20,21]$ and the results are compiled in Figures 2 in the case of constant material properties and in Figures 3(a) and 3(b) when the material properties depend on concentrations (numerical values of these material properties are also tabulated in Appendix A). This data allows us to assert that the results obtained with inverse modelling and the Planck-Nernst model modified to account for the dendritic growth do indeed fall in the correct range. Finally, we will use the recently developed tools of Bayesian inference [38] to blend the results from the literature together with our experimental data and the assumed mathematical model to quantify the uncertainty of the reconstructed material properties.

The structure of the paper is as follows: in the next section we describe our experimental set-up, in Section 3 the Planck-Nernst model (1) is modified to account for the dendrite growth; our computational approach is then described in Section 4 and the main results are presented in Section 5; discussion and final conclusions are deferred to Section 6. Supplementary data and the technical background are presented in three appendices.

\section{Experimental}

The experiment monitors the gradual build-up of the ionic concentration gradient in an electrolyte solution which results from the application of a constant current, starting from an initially uniform concentration throughout the solution volume. It is carried out under galvanostatic conditions in a symmetric Li-Li electrochemical cell constructed from a NMR tube with $4.2 \mathrm{~mm}$ inner diameter and $20 \mathrm{~mm}$ inter-electrode distance (see Figure 4), filled with a $1 \mathrm{M} \mathrm{LiPF}_{6}$ solution in a 1:1 binary mixture of Ethylene Carbonate (EC) and Di-Methyl Carbonate (DMC). A constant current of 75 $\mu \mathrm{A}$ (corresponding to a current density of $541.38 \mu \mathrm{A} \mathrm{cm} \mathrm{cm}^{-2}$ ) was applied to the cell for 14 hours. Concentration profiles were acquired using magnetic resonance imaging (MRI). For this experiment we chose to monitor the ${ }^{19} \mathrm{~F}$ nuclei, which significantly reduces the data acquisition time, since the relative NMR sensitivity to a ${ }^{19} \mathrm{~F}$ nucleus is approximately 3 times higher than to a ${ }^{7} \mathrm{Li}$ nucleus. Onedimensional ${ }^{19} \mathrm{~F}$ NMR images were obtained using a gradient spin-echo pulse sequence with the magnetic field gradient applied along the static magnetic field $B_{0}$ (i.e., along the axis of the cell), with a $3 \mathrm{~ms}$ echo time and a $20 \mathrm{G} / \mathrm{cm}$ reading gradient [12]. Over the course of the experiment 256 frequencydomain points were collected over the spectral width of $200 \mathrm{kHz}$. The combination of the magnetic field gradient and spectral resolution yielded a spatial resolution of $40 \mu \mathrm{m}$. A total of 64 scans with a relaxation delay of $3.5 \mathrm{~s}$ were collected for each image, resulting in an acquisition time of 4 minutes per image. The imaging measurement sequence was repeated at 2-hour intervals uniformly spread over 14 hours duration of the galvanostatic experiment. The experimentally obtained concentration profiles, hereafter denoted $\tilde{c}(x, t)$, are shown in Figure 4 at different times $t \in[0,14$ hours $]$ as functions of the space coordinate $x$. It is known that at the microstructure level complex dendritic structures have distinct growth spots [42] and the Li concentration at the solid-liquid interfaces in these growths spots drops to zero [10] (although other mechanisms have also been reported, e.g., in [6]). The reason why this effect is not evident in Figure 4 is because the profiles in this plot represent concentrations averaged 
over the entire cross-section of the cell. The growth of the dendritic region is modelled based on a separate experiment designed to probe how this region expands with time, cf. figure 1(b), depending on the applied current $I$ (we add that at the end of the experiment the dendritic region could be visually observed in the cell). These results are presented in Figure 5 where we show the position $l(t)$ of the left boundary of the dendritic region in function of time $t$. As is evident from this data, there is an approximately linear dependence of $l(t)$ on time with the magnitude of the proportionality constant (corresponding to the slope the linear fits to the data in Figure 5) increasing with the applied current. This observation together with the data from Figure 4 allows us to quantify the growth of the dendritic region $[l(t), L]$, which will be used in developing a modified Planck-Nernst model that accounts for the effects of dendritic growth in the next section.

\section{The Planck-Nernst Model with Dendrite Growth}

Based on the observations made in Section 2 about the growth of the dendritic region, we now propose a modification of the Planck-Nernst model (1) that accounts for this phenomenon. We emphasize that this is an idealized model which aims to describe the depletion of lithium from the electrolyte in the dendritic region without attempting to provide detailed information about the structure of this region. In formulating this modified model we retain assumptions A1-A7 from Section 1, replace assumption A8 with a new assumption A8' given below and add the following assumptions A9-A11

A8': lithium ions are stripped from the anode (at $x=0)$ uniformly in the transverse direction and their flux corresponds to the applied electric current [31, 34];

A9: lithium ions are deposited on the cathode non uniformly in space forming a spatially distributed dendritic structure that grows into the electrolyte at a rate proportional to the applied current [48]; the domain occupied by the dendrites is $[l(t), L]$, cf. Figure $1(\mathrm{~b})$, where $l(t)$ is a known decreasing function of time such that $l(0)=L$, cf. Figure 5 ;

A10: Throughout the experiment the dendrites occupy a negligible fraction of the volume of the electrolyte such that porosity of this region need not be taken into account;

A11: The loss of cations from the electrolyte due to their deposition on the dendrites is modeled by a sink term with a prescribed spatial distribution $\mathcal{F}_{l(t)}(x)$ that is zero outside the dendritic region $[l(t), L]$; this sink term, which has units of $\mathrm{m}^{-1}$, should be understood as "effective", in the sense that it represents the number of $\mathrm{Li}^{+}$ions that are transferred onto the surface of the dendrite structure per unit volume of the electrolyte/dendrite region.

With these additional assumptions, our model now takes the form

$$
\begin{aligned}
\frac{\partial c}{\partial t} & =\frac{\partial}{\partial x}\left[D \frac{\partial c}{\partial x}+\frac{\left(1-t^{+}\right) I}{F A}\right]-\frac{\left(1-t^{+}\right) I}{F A} \mathcal{F}_{l(t)}(x) & & \text { in }(0, L) \times(0, T], \\
\left.\frac{\partial c}{\partial x}\right|_{x=0} & =-\frac{\left(1-t^{+}\right) I}{D F A} & & \text { in }(0, T], \\
\left.\frac{\partial c}{\partial x}\right|_{x=L(0)} & =0 & & \text { in }(0, T], \\
\left.c\right|_{t=0} & =c_{i} & & \text { in }(0, L),
\end{aligned}
$$

where $l(t)=L-k I t$ with a certain constant $k>0$, which is justified by the data presented in Figure 5 . We note that the value of the constant $k$, which describes how rapidly the dendritic region $[l(t), L]$ spreads into the bulk of the electrolyte, can be deduced from the concentration measurements shown in Figure 4 by assessing the width $L-l(T)$ of the dendritic region at the end of the experiment. The key difference between the standard Planck-Nernst model (1) and its modified version (2) is the presence 
of the sink term proportional to the function $\mathcal{F}_{l(t)}(x)$ which describes how lithium from the electrolyte is lost due to its deposition within the growing dendritic region $[l(t), L]$. While this process can be described in various ways, the proposed model (2a) combines simplicity with the flexibility inherent in allowing different possible forms for the function $\mathcal{F}_{l(t)}(x)$, which we will refer to as the "sink function". However, this model is only effective, in the sense that it makes no attempt to capture the complex physics of the dendrite-formation process. Given the different lithium deposition scenarios evidenced in the experimental studies discussed in the Introduction, we will consider the following three distinct forms of "sink function":

case A: loss of lithium ions from the electrolyte occurs only at the interface $x=l(t)$ between the bulk of the electrolyte and the dendritic region, i.e.,

$$
\mathcal{F}_{l(t)}(x)=\delta(l(t)-x),
$$

where $\delta(\cdot)$ is the Dirac distribution; this corresponds to deposition occurring at the tips of the growing dendrites only,

case B: the rate of lithium ion loss from the electrolyte decreases linearly from its maximum at the moving interface $(x=l(t))$ to zero at the electrode $(x=L)$, i.e.,

$$
\mathcal{F}_{l(t)}(x)=\left\{\begin{array}{ll}
\frac{2(L-x)}{(L-l(t))^{2}} & l(t) \leq x \leq L \\
0 & \text { otherwise }
\end{array},\right.
$$

case $\mathrm{C}$ : the rate of lithium ion loss from the electrolyte is uniform between the moving interface at $x=l(t)$ and the electrode at $x=L$, i.e.,

$$
\mathcal{F}_{l(t)}(x)=\left\{\begin{array}{ll}
\frac{1}{L-l(t)} & l(t) \leq x \leq L \\
0 & \text { otherwise }
\end{array} .\right.
$$

We note that cases $\mathrm{A}, \mathrm{B}$ and $\mathrm{C}$ correspond to the rate of the loss of lithium being progressively less localized near the tips of the dendrites at $x=l(t)$, and in all cases the sink function is normalized such

that $\int_{l(t)}^{L} \mathcal{F}_{l(t)}\left(x^{\prime}\right) d x^{\prime}=1$. Moreover, we emphasize that a model in which the dendrite growth occurs from the base of the structure, at $x=L$, corresponds to taking $\mathcal{F}_{l(t)}(x)=\delta(L-x)$ and that this is in fact equivalent to the original, unmodified, model (1), and so we do not consider this case further.

\section{Computational Approaches to Inverse Modelling and Uncer- tainty Quantification}

In this section we provide an overview of the computational approaches employed to solve the inversemodelling problem for both constant and concentration dependent material properties and assess their uncertainty. Further details are provided in Appendices B and C.

\subsection{Inverse Modelling}

The unknown material properties, $D$ and $t^{+}$, can be reconstructed based on the assumed transport model, such as system (1) or (2), and using the concentration profiles obtained in the NMR experiment described in Section 2. We will use the inverse modelling approach developed and validated in [40] in which the problem is framed as minimization of a cost functional representing the least-squares deviation between $c$, the concentration profiles predicted by the model (1)-(2), and $\tilde{c}$, the experimentally determined concentration profiles. The cost functional can thus be represented in the form

$$
\mathcal{J}\left(\left[D, t^{+}\right]\right)=\frac{1}{2} \sum_{i=1}^{N_{T}} \int_{0}^{L}\left[c\left(x, t_{i} ; D, t^{+}\right)-\tilde{c}\left(x, t_{i}\right)\right]^{2} d x,
$$


where $t_{i}, i=1, \ldots, N_{T}$, are the time levels when the experimental concentration profiles are acquired $\left(N_{T}=14\right.$, cf. Section 2).

We consider two distinct formulations corresponding, respectively, to constant and to concentrationdependent material properties. The first of these pertains to the standard Nernst-Planck theory, while the second of these represents the extension discussed in [40]. Henceforth we distinguish between the two cases by using the notations: $D$ and $t^{+}$for the standard Nernst-Planck theory and $D(c)$ and $t^{+}(c)$ for the extension to this theory.

When both $D$ and $t^{+}$are constant, we obtain a simple optimization problem (which is exact in the limiting case of an ideal solution, i.e., at very dilute salt concentrations)

$$
\mathrm{P} 1: \quad\left[\widehat{D}, \widehat{t}^{+}\right]=\underset{\left[t^{+}, D\right] \in \mathbb{R}^{2}}{\operatorname{argmin}} \mathcal{J}\left(D, t^{+}\right)
$$

(henceforth carets " $r$ " will denote optimal reconstructions). Problem P1 is rather well understood and can be solved in a straightforward manner using commercially available software tools such as the minimization routines in MATLAB. It was in fact already solved in the seminal study by Klett et al. [20] and is also solved here as a preliminary step in a more complete analysis.

The more complete optimization problem, which is better able to reflect the physics of the problem, arises when both $D(c)$ and $t^{+}(c)$ are allowed to depend on the ion concentration $c$; it is

$$
\mathrm{P} 2: \quad\left[\widehat{D}(c), \widehat{t}^{+}(c)\right]=\underset{\left[t^{+}(c), D(c)\right] \in \mathcal{X}}{\operatorname{argmin}} \mathcal{J}\left(D(c), t^{+}(c)\right),
$$

where $\mathcal{X}$ denotes a suitable function space to which $D(c)$ and $t^{+}(c)$ belong. We emphasize that, apart from smoothness and the limiting behavior for large and small values of $c$, no other a priori assumptions are made about the functional forms of $D(c)$ and $t^{+}(c)$. In contrast to the simplified problem $\mathrm{P} 1$, the computational approach required to solve the more realistic problem P2 with concentration-dependent material properties is more involved and necessitates specialized tools described in detail in Appendix B.

\subsection{Bayesian Uncertainty Quantification}

In order to quantify the uncertainty arising in estimation due to, e.g., modelling and measurement errors, we use a state-of-the-art technique based on Bayesian inference [38]. In this approach a probabilistic setting is adopted as a way to quantify uncertainty resulting from incomplete knowledge about the problem. When our measurements are incomplete and also inaccurate (e.g., due to noise), then an inverse problem may admit many approximate solutions. Bayesian inference is an elegant and consistent framework allowing one to use a combination of prior knowledge and experimental data in order to assign specific confidence to different reconstructions of the material properties. Therefore, here we will represent the reconstructed material properties $\left[D, t^{+}\right]$, or $\left[D(c), t^{+}(c)\right]$, in terms of random variables characterized by certain probability density functions (PDFs). More precisely, in the case of concentration-dependent properties, $D(c)$ and $t^{+}(c)$ are given by suitable probability distributions for all concentration values $c \in\left[c_{\alpha}, c_{\beta}\right]$ and the same also applies to the measurements $\tilde{c}$ for different values of $x \in[0, L]$ and $t \in[0, T]$.

For brevity, here we will describe our approach to uncertainty quantification for the case of constant material properties $\left[D, t^{+}\right]$and refer the reader to [38] for details of the technically more involved problem when the material properties depend on the concentrations $\left[D(c), t^{+}(c)\right]$. In the Bayesian framework the probability distribution of the reconstructed material properties is given in terms of the posterior probability of the diffusion and transference numbers taking values $\mathbb{P}\left(\left[D, t^{+}\right] \mid \tilde{c}\right)$ given the entire set of observations $\tilde{c}$. This can be expressed using Bayes' Theorem, $\mathbb{P}(A \mid B) \mathbb{P}(B)=\mathbb{P}(B \mid A) \mathbb{P}(A)$, where $A$ and $B$ are events $[41,45,46]$, in the form

$$
\mathbb{P}\left(\left[D, t^{+}\right] \mid \tilde{c}\right)=\frac{\mathbb{P}\left(\tilde{c} \mid\left[D, t^{+}\right]\right) \mathbb{P}\left(\left[D, t^{+}\right]\right)}{\mathbb{P}(\tilde{c})} .
$$


Here $\mathbb{P}\left(\left[D, t^{+}\right]\right)$is the prior distribution and reflects our a priori assumptions about the solution; in practice it is based on previous studies in the literature estimating the diffusivity $D$ and transference number $t^{+}$. Computation of the prior $\mathbb{P}\left(\left[D, t^{+}\right]\right)$is described below. The term $\mathbb{P}\left(\tilde{c} \mid\left[D, t^{+}\right]\right)$is the likelihood of observing particular experimental data $\tilde{c}$ for a given set of material properties $\left[D, t^{+}\right]$, while $\mathbb{P}(\tilde{c})$ is the overall probability of observing the experimental data $\tilde{c}$ and can be treated as a normalizing factor.

In the present study the prior $\mathbb{P}\left(\left[D, t^{+}\right]\right)$is constructed using the data reported in the literature for the diffusivity and the transference numbers for the electrolyte considered here, cf. Figures 2 and 3 as well as Appendix A, and is shown in Figure 6. Details concerning the computation of such a prior are provided in Appendix C. Priors defined in this way can be "weak" or "strong" [23], depending on their relative deviation from the uniform distribution which is also reflected in the width of the range $\left[\min _{D, t^{+}} \mathbb{P}\left(\left[D, t^{+}\right]\right), \max _{D, t^{+}} \mathbb{P}\left(\left[D, t^{+}\right]\right)\right]$. Given the differences in experimental conditions resulting in various possible interpretations of the data from the literature, the prior adopted in the present study is "weak", cf. Figure 6, such that it will not dominate the posterior probability given in (7).

As regards the likelihood function, the following ansatz is typically adopted in Bayesian inference $[41,45,46]$

$$
\mathbb{P}\left(\tilde{c} \mid\left[D, t^{+}\right]\right) \propto e^{-\mathcal{J}\left(D, t^{+}\right)},
$$

where $\mathcal{J}\left(D, t^{+}\right)$is the error functional defined in (6). This expresses the assumption that for a given set of material properties $\left[D, t^{+}\right]$, measurements resulting in large values of the error functional are less likely to be observed. An intuitive motivation for the choice of an exponential function in (8) is that in the hypothetical simplified case when the predicted concentrations have a linear dependence on the material properties, resulting in $\mathcal{J}$ being a quadratic function of the material properties, the relation (8) would produce a normal distribution which in the light of the central limit theorem is universal. A more rigorous justification of this choice can be found for example in [45]. The likelihood function $\mathbb{P}\left(\tilde{c} \mid\left[D, t^{+}\right]\right)$is approximated by sampling the distribution in (8) using the Metropolis-Hastings algorithm [11] to produce $M$ samples. Details concerning the computational algorithm are provided in Appendix C. The normalizing factor $\mathbb{P}(\tilde{c})$ in $(7)$ is determined such that the integral of the posterior probability $\mathbb{P}\left(\left[D, t^{+}\right] \mid \tilde{c}\right)$ with respect to $D$ and $t^{+}$is equal to unity, which explains the rather large values attained by the posterior probability, cf. Figures 7 and 12 below (the characteristic values of the diffusivity $D$ are $\left.\mathcal{O}\left(10^{-11}\right)\right)$.

Thus, the Bayesian representation of the uncertainty (7) combines the knowledge about the material properties already available in the literature as the prior $\mathbb{P}\left(\left[D, t^{+}\right]\right)$, cf. Figure 6 , with a measure of uncertainty based on how well model (2) fits the data which is represented by the likelihood function $\mathbb{P}\left(\tilde{c} \mid\left[D, t^{+}\right]\right)$. Since a weak prior is used, the posterior distribution $\mathbb{P}\left(\left[D, t^{+}\right] \mid \tilde{c}\right)$ is slightly skewed in favor of the likelihood function. This approach to uncertainty quantification in electrochemical systems was developed and thoroughly validated in [38].

\section{$5 \quad$ Results}

In this section we present and analyze the reconstructions of the material properties obtained using the inverse-modelling approach of Section 4.1 based on the standard and modified Planck-Nernst models, (1) and (2). The uncertainty of the obtained estimates is quantified with the Bayesian approach described in Section 4.2. First, we focus on inferring constant material properties by solving problem P1, and then consider reconstruction of concentration-dependent material properties by solving problem P2 based on the modified model accounting for dendrite growth (2). We emphasize that these material properties are optimal since they minimize the corresponding error functionals.

We begin the presentation of our results by showing the optimal reconstructions $\widehat{D}$ and $\widehat{t}^{+}$of the constant material properties, via problem P1, based first on the standard Planck-Nernst model (1) and then based on the modified model accounting for dendritic growth (2) in which the three different forms of distributed sink function $\mathcal{F}_{l(t)}(x)$ postulated in $(3)-(5)$ are employed. These reconstructions are shown 
in Figure 7. The reconstructions are obtained by solving optimization problem P1 and the corresponding values of the error functional (6) are given in Table 1. Figure 7 also contains information about the relative uncertainty of the reconstructions determined as discussed in Section 4.2. More specifically, in each panel of Figure 7 the intersection of the solid lines represent the optimal reconstructions $\left[\widehat{D}, \hat{t}^{+}\right]$ obtained by solving minimization problem $\mathrm{P} 1$, while the color contours represent the corresponding posterior probability distributions (7). The data shown in Figure 7(a) confirms the observations already made in [36], namely, that the standard Planck-Nernst model (1) leads to a negative transference number $\widehat{t}^{+}<0$ obtained via inverse modelling, which is physically inconsistent and hence calls into question the validity of the direct application of the standard Planck-Nernst system (1) to model the data described in Section 2. This issue is only partially remedied, in the sense that the obtained values of $\widehat{t}^{+}$become less negative, when the reconstructions are performed based on the modified model (2) with the sink function $\mathcal{F}_{l(t)}(x)$ defined in $(3)$ and $(4)$, cf. Figures $7(\mathrm{~b})$ and $7(\mathrm{c})$. We note that in all these cases the maximum of the posterior probability distribution $\mathbb{P}\left(\left[D, t^{+}\right] \mid \tilde{c}\right)$ corresponds to positive transference number $t^{+}$, which is the effect of the prior obtained based on positive transference numbers only, cf. Figure 6. On the other hand, in Figure 7(d) we observe that the reconstructed transference number $\widehat{t}^{+}$obtained based on the modified Planck-Nernst model (2) with a uniform sink function (5) is positive. In this case the optimal reconstructions $\left[\widehat{D}, \hat{t}^{+}\right]$are also near the values of $\left[D, t^{+}\right]$maximizing the posterior probability distribution $\mathbb{P}\left(\left[D, t^{+}\right] \mid \tilde{c}\right)$, indicating that the reconstructions $\left[\widehat{D}, \widehat{t}^{+}\right]$are now closer to the literature data. Moreover, since the posterior probability distribution in Figure $7(\mathrm{~d})$ is more isotropic than in the previous cases, this implies a weaker correlation between the uncertainties of $D$ and $t^{+}$. By comparing the results shown in Figures 7(a)-(d) we note that the main improvement, both in terms of the reconstructed values and their uncertainties, results from replacing models with a localized depletion of lithium (i.e., the standard Planck-Nernst model with the boundary condition (1b) and the modified Planck-Nernst model (2) with the sink function given in case A) with models in which this depletion is distributed in space (the modified Planck-Nernst model (2) with the sink functions given in cases B and C). In order to shed additional light on how the structure of the sink function affects the reconstruction of the transference number $t^{+}$, we have solved problem P1 using a family of sink function constructed such that the constant "slope" $s=\frac{d}{d x} \mathcal{F}_{l(t)}(x), x \in[l(t), L]$, was allowed to vary continuously from $-\infty$ to $+\infty$ while satisfying the constraint $\int_{l(t)}^{L} \mathcal{F}_{l(t)}\left(x^{\prime}\right) d x^{\prime}=1$. Thus, the standard Planck-Nernst model (1) and variants A-C of the modified model (2), cf. (3)-(5), can be viewed as special cases of this more general family, see Figure 8(a). As is evident from the data shown in Figure 8(b), within this family of sink functions, case $\mathrm{C}$ corresponds in fact to the maximum value of the reconstructed transference number $\widehat{t}^{+}$.

The experimental concentration profiles $\tilde{c}\left(x, t_{i}\right)$ are compared to the concentration profiles $c\left(x, t_{i}\right)$ predicted by the standard Planck-Nernst model (1) and the modified model (2) with a uniform sink function (5) using their respective optimal material properties $\left[\widehat{D}, \widehat{t}^{+}\right]$in Figures 9-10 (left and middle columns). The main difference between these two cases is that in the former the concentration profiles $c\left(x, t_{i}\right)$ predicted by the model exhibit large deviations from the measured profiles $\tilde{c}\left(x, t_{i}\right)$ close to the right electrode, which is where the dendritic regions appears. We remark that, as discussed in Section 2 , these concentration values are averaged over the cross-section of the cell and may therefore involve growth spots where the concentration locally drops to zero [10,42]. The quality of fits in this region is noticeably improved when the modified Planck-Nernst model (2) is used, arguably due to a suitable choice of the sink function $\mathcal{F}_{l(t)}(x)$, cf. (5). This improvement is also reflected in the difference of the values of the error functional (6) obtained in the two cases, cf. Table 1. As deposition of lithium continues, a porous structure with a certain porosity $\phi(x, t)$ depending on both space and time is formed in the dendritic region $[l(t), L]$. The corresponding volume fractions $1-\phi(x, t)$ occupied by lithium dendrites predicted by the modified Planck-Nernst model (2) with a uniform sink function (5) are shown in Figure 11 for as functions of $x \in[l(T), L]$ for different times spread uniformly between 0 and $T$. This data illustrates how the dendritic region progressively spreads into the bulk of the electrolyte. Another interpretation is that if one assumed that all lithium is deposited on a single dendrite, then the curves shown in Figure 11 would describe its time-evolving shape. The average porosity of the dendritic 
region at the end of the experiment is $\left[\int_{l(T)}^{L} \phi(x, T) d x\right] /[L-l(T)]=0.9675$, retrospectively justifying our assumption A10 in Section 3.

Since it gives the best results, we now focus on the modified Planck-Nernst model (2) with a uniform sink function (5) and consider reconstruction of concentration-dependent material properties by solving problem P2. The optimal reconstructions $\widehat{D}(c)$ and $\widehat{t}^{+}(c)$ are shown as functions of the concentration $c$ in Figures 12(a) and 12(b), respectively, together with the associated posterior probability distributions. As is evident from these figures, the obtained optimal reconstructions $\widehat{D}(c)$ and $\widehat{t}^{+}(c)$ are consistent, both in terms of values attained and the dependence on $c$, with the literature data summarized in Figures $3(\mathrm{a})$ and $3(\mathrm{~b})$, although the literature data for the transference numbers exhibits a significant scatter for all values of $c$. We note that the uncertainty of $\widehat{D}(c)$ and $\widehat{t}^{+}(c)$, given by the posterior probability distribution at the given value of $c$, is rather modest and significantly smaller than the entire range of variation of $\widehat{D}(c)$ and $\widehat{t}^{+}(c)$ for $c \in[200,2000]$. This uncertainty is also essentially independent of the concentration $c$. Finally, in Figures 9-10 (right columns) we compare the corresponding concentration profiles $c\left(x, t_{i}\right)$ with the experimental profiles $\tilde{c}\left(x, t_{i}\right)$. As is evident from this comparison, there is further improvement in the quality of the fits, which is also reflected in an additional decrease of the value of the error function (6), cf. Table 1.

\section{Conclusions}

In this section we briefly summarize our findings from Section 5. As is evident from Figure 7(d), the modified Planck-Nernst model (2) with a uniform sink function (5) leads to the most consistent reconstruction of the material properties, since in all other cases, including the standard Planck-Nernst model (1), the inferred transference numbers $\widehat{t}^{+}$are negative, cf. Figures $7(\mathrm{a})-(\mathrm{c})$. This demonstrates that the uniform sink function (5), describing a uniform in space depletion of lithium, offers a satisfactory account of the effect of the formation of the dendritic region on the transport processes in the electrolyte (we have also considered forms of the sink function $\mathcal{F}_{l(t)}(x)$ other than given in (3)-(4), but the results were inferior with respect to those obtained with (5)). This conclusion is reinforced by the improved quality of the concentration fits near the right electrode obtained with the modified model (2) as compared to the fits obtained with the standard model (1), cf. Figures 9-10 (left versus middle and right columns). The fact that the modified model (2) reproduces the experimental measurements most accurately when it is combined with a uniform sink function (5) allows us to speculate that lithium is deposited not only on the tips of the dendrites, but also within the entire dendritic region (for example, in the form of new dendrites growing from the negative electrode or sideways off the existing dendrites). Modelling this process based on first principles remain a challenging open problem [48].

The reconstructions obtained based on the modified model (2) with the uniform sink function (5) are characterized by the smallest uncertainty with the weakest degree of correlation between the uncertainties of $D$ and $t^{+}$. Finally, we also note that the concentration-dependent diffusivity $\widehat{D}(c)$ reconstructed by solving problem P2 agrees well with the literature data, both in terms of the values and the dependence on $c$, cf. Figures 3(a) and 12(a). As regards the concentration-dependent transference numbers, the obtained reconstruction $\widehat{t}^{+}(c)$ corresponds to the lower end of the range of values reported in the literature, cf. Figures 3(b) and 12(b). Concerning the dependence on the concentration, the decrease of the optimally reconstructed material property $\widehat{t}^{+}(c)$ with $c$ is consistent with the trends evident in most, albeit not in all, datasets from the literature.

To conclude, we have developed and validated a novel model for the transport of charged species in electrolytes in the presence of dendritic growth. We also add that the present study represents an innovative application of the concepts of inverse modelling and uncertainty quantification to validate or invalidate different models of complex transport phenomena. 


\section{Acknowledgments}

The authors are thankful to Dr. I. C. Halalay for helpful discussions. Funding for this research was provided by an NSERC (Canada) Strategic Project Grant (\# STPGP 479258-15). GR was supported by the Faraday Institution Multi-Scale Modelling (MSM) project Grant number EP/S003053/1. 


\section{A Values of Material Properties Reported in the Literature}

- Y. Aihara et al., 2004 [1]

\begin{tabular}{l||llll}
\hline$c\left[\mathrm{~mol} / \mathrm{m}^{3}\right]$ & 100 & 250 & 500 & 750 \\
\hline$D\left[\mathrm{~m}^{2} / \mathrm{s}\right]$ & 3.5 & 3.1 & 2.5 & 2.1 \\
$t^{+}$ & 0.33 & 0.35 & 0.34 & 0.34 \\
\hline
\end{tabular}

- K. Hayamizy, 2012 [17]

$$
\begin{array}{l||l}
\hline D\left[\mathrm{~m}^{2} / \mathrm{s}\right] & 1.41 \\
\hline
\end{array}
$$

- M. Klett et al., 2013 [20]

\begin{tabular}{l||l}
\hline$D\left[\mathrm{~m}^{2} / \mathrm{s}\right]$ & 1.1 \\
\hline$t^{+}$ & 0.33 \\
\hline
\end{tabular}

- K. Kondo et al., 2000 [21]

\begin{tabular}{l||lllllllll}
\hline$c\left[\mathrm{~mol} / \mathrm{m}^{3}\right]$ & 1 & 118 & 565 & 1100 & 1600 & 2070 & 2500 & 2910 & 3290 \\
\hline$D\left[\mathrm{~m}^{2} / \mathrm{s}\right]$ & 4.9 & 4.2 & 2.7 & 1.4 & 0.74 & 0.37 & 0.17 & 0.090 & 0.061 \\
\hline
\end{tabular}

- S. Krachkovskiy et al., 2013 [22]

\begin{tabular}{l||llll}
\hline$c\left[\mathrm{~mol} / \mathrm{m}^{3}\right]$ & 200 & 800 & 1200 & 1800 \\
\hline$D\left[\mathrm{~m}^{2} / \mathrm{s}\right]$ & 2.13 & 1.11 & 0.82 & 0.45 \\
$t^{+}$ & 0.38 & 0.39 & 0.39 & 0.45 \\
\hline
\end{tabular}

- A. Mehrotra et al., 2013 [26]

\begin{tabular}{l||llll}
\hline$c\left[\mathrm{~mol} / \mathrm{m}^{3}\right]$ & 200 & 400 & 600 & 800 \\
\hline$D\left[\mathrm{~m}^{2} / \mathrm{s}\right]$ & 7.11 & 6.54 & 3.21 & 2.14 \\
\hline \hline$t^{+}$ & 0.41 & & & \\
\hline
\end{tabular}

- J. Newman et al., 2003 [30]

\begin{tabular}{l||l}
\hline$D\left[\mathrm{~mol} / \mathrm{m}^{3}\right]$ & 0.27 \\
\hline$t^{+}$ & 0.5 \\
\hline
\end{tabular}

- M. Riley et al., 2002 [37]

\begin{tabular}{l||llllll}
\hline$c\left[\mathrm{~mol} / \mathrm{m}^{3}\right]$ & 100 & 250 & 500 & 750 & 1000 & 1500 \\
\hline$t^{+}$ & 0.3 & 0.22 & 0.18 & 0.12 & 0.10 & 0.01 \\
\hline
\end{tabular}

- S. Stewart et al., 2008 [44]

\begin{tabular}{l||l}
\hline$D\left[\mathrm{~mol} / \mathrm{m}^{3}\right]$ & 0.77 \\
\hline$t^{+}$ & 0.4 \\
\hline
\end{tabular}

- L. Valoen et al., 2005 [47]

\begin{tabular}{|c|c|c|c|c|}
\hline$c\left[\mathrm{~mol} / \mathrm{m}^{3}\right]$ & 0 & 1000 & 1750 & 2250 \\
\hline $\mathrm{t}^{+}$ & 0.4 & 0.36 & 0.39 & 0.37 \\
\hline
\end{tabular}

- J. Zhao et al., 2008 [52]

\begin{tabular}{l||lllll}
\hline$c\left[\mathrm{~mol} / \mathrm{m}^{3}\right]$ & 250 & 500 & 750 & 1000 & 1500 \\
\hline$D\left[\mathrm{~m}^{2} / \mathrm{s}\right]$ & 2.14 & 1.88 & 2.23 & 1.70 & 1.42 \\
$t^{+}$ & 0.557 & 0.477 & 0.445 & 0.408 & 0.370 \\
\hline
\end{tabular}

- S. Zugmann et al., 2011 [53]

\begin{tabular}{l||l}
\hline$D\left[\mathrm{~mol} / \mathrm{m}^{3}\right]$ & 3.37 \\
\hline$t^{+}$ & 0.24 \\
\hline
\end{tabular}




\section{B Gradient-Based Approach to Solution of Optimization Prob- lem P2}

An iterative optimization algorithm to reconstruct concentration-dependent material properties $D(c)$ and $t^{+}(c)$ based on a simple gradient descent approach can be written as

$$
\begin{aligned}
D^{(n+1)}(c) & =D^{(n)}(c)-\xi_{D}^{(n)} \nabla_{D} \mathcal{J}\left(D^{(n)}(c), t^{+(n)}(c)\right) & & n=1,2, \ldots, \\
t^{+(n+1)}(c) & =t^{+(n)}(c)-\xi_{t^{+}}^{(n)} \nabla_{t^{+}} \mathcal{J}\left(D^{(n+1)}(c), t^{+(n)}(c)\right) & & n=1,2, \ldots, \\
{\left[D^{(0)}(c), t^{+(0)}(c)\right] } & =\left[\widehat{D}, \widehat{t}^{+}\right], & &
\end{aligned}
$$

where $\xi_{D}^{(n)}$ and $\xi_{t^{+}}^{(n)}$ are the step sizes in the direction of the gradients. A key element of this algorithm is computation of the gradients $\nabla_{D} \mathcal{J}$ and $\nabla_{t^{+}} \mathcal{J}$ of the cost functional (6), which are functions of the concentration $c$. They can be efficiently evaluated by solving suitably-defined "adjoint problems", which are system of partial differential equations encoding information about the sensitivity of the solutions to the governing system (1) or (2) to infinitesimal perturbations of the material properties $D(c)$ and $t^{+}(c)$. The governing and the adjoint system are solved using MATLAB's inbuilt function pdepe which performs an adaptive discretization both in space and time of a specified partial differential equation. The relative and absolute tolerance used in the solution process were $10^{-8}$ and $10^{-10}$, respectively. The concentration-dependent material properties are discretized using 300 equispaced points and a suitable interpolation function is used in pdepe to obtain values of the material properties at different points in space and in time. The gradients are then made sufficiently smooth, as dictated by the required regularity of the functions $D(c)$ and $t^{+}(c)$. Once the gradients are thus determined, the step sizes $\xi_{D}^{(n)}$ and $\xi_{t^{+}}^{(n)}$ are computed by solving line-minimization problems [33]. The initial guess (9c) is given by the constant material properties obtained as the solution of problem P1, however, our computational experiments indicate that the optimal reconstructions $\widehat{D}(c)$ and $\widehat{t}^{+}(c)$ are not very sensitive to the initial guess. We add that, as discussed in [39], problem P1 was in fact found to admit global minima, meaning that in that problem convergence is achieved regardless of the initial guess. We refer the reader to [40] for all derivation details in the context of the Planck-Nernst system and to [7, 8] for a discussion of the mathematical foundations of this approach, which is summarized as Algorithm 1. In actual computation calculation are accelerated with the use of the conjugate gradients method [33].

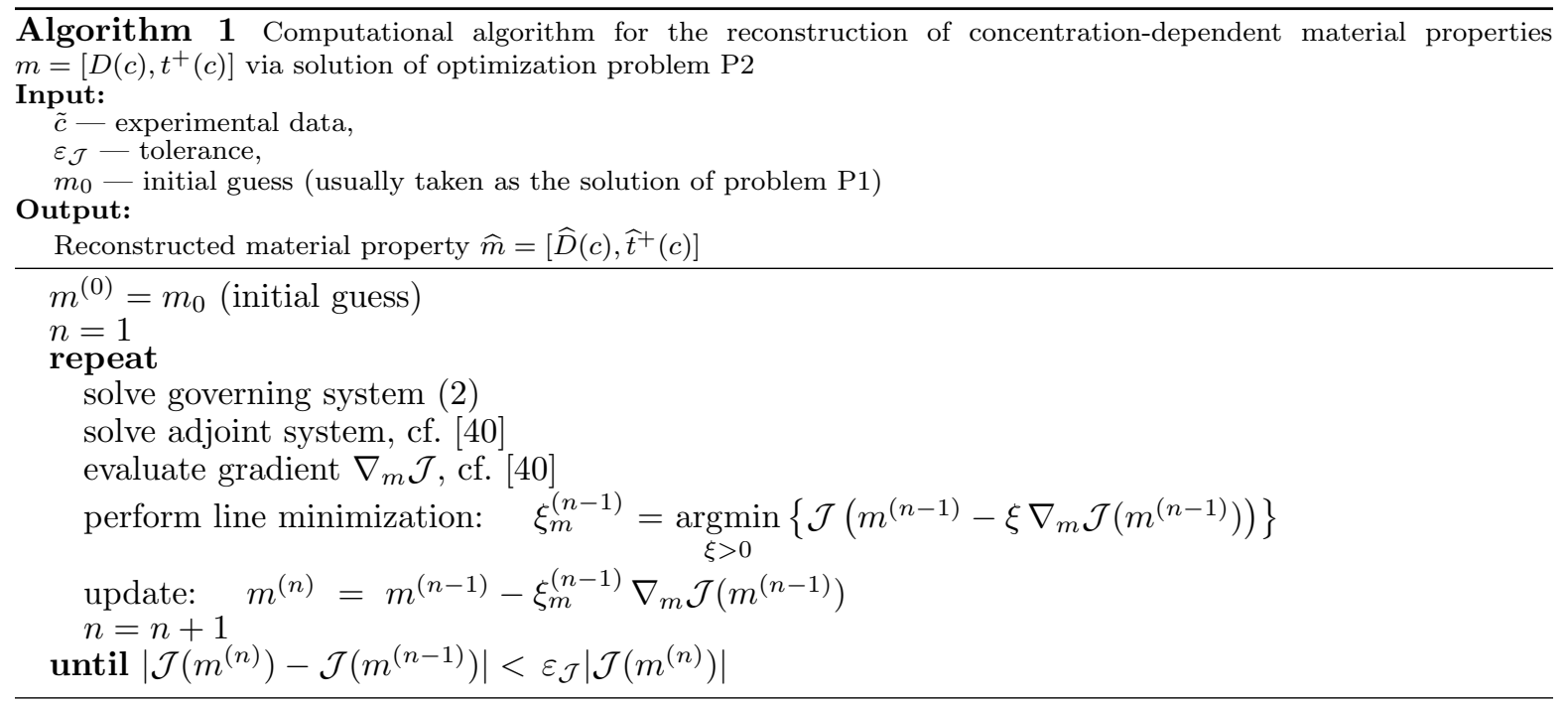




\section{Determination of the Prior and Sampling the Likelihood Function}

The prior $\mathbb{P}\left(\left[D, t^{+}\right]\right)$in Figure 6 is constructed based on the literature data for the diffusivity and the transference numbers for the electrolyte considered in our study, which is summarized in Figures 2 and 3, and is also tabulated in Appendix A. This is done using the function kde2d from the MASS package in $\mathrm{R}$, where the bandwidth and the number of grid points are suitably adjusted to obtain a prior with the desired "weakness".

Sampling the likelihood function (8) is performed using the Metropolis-Hastings algorithm [11]. For brevity of notation, we will denote $m=\left[D, t^{+}\right]$. This algorithm is based on the Markov-Chain Monte-Carlo (MCMC) approach [16] employed to randomize $m$ and at each step involves solution of the governing system (1) or (2) for modified (trial) material properties $m^{(*)}$ followed by the evaluation of the error functional (6). At each step the algorithm explores the probability space by collecting samples from the probability distribution (7). In problem P1 this is done simply by adding a random vector sampled from the normal distribution with zero mean and standard deviations given by $\left[C_{D}, C_{t^{+}}\right]$ for the two material properties. On the other hand, in problem P2 a move in the probability space is performed in terms of the Fourier-space representation of the concentration-dependent material properties computed using the cosine transform (the choice of the cosine-series representation is dictated by the assumed behavior of the reconstructed material properties $\widehat{D}(c)$ and $\left.\widehat{t}^{+}(c)\right]$ at the endpoints of their domain of definition, i.e., for small and large concentrations values [38]). The individual cosine-series coefficients are then multiplied by random numbers drawn from the normal distribution with unit mean and standard deviation $C$. Formulation of this step in Fourier space ensures that randomized material properties retain the required smoothness as functions of the concentration $c$, i.e., they remain in the space $\mathcal{X}$, cf. problem P2. In either case, a move in the probability space is accepted or rejected based on a sample acceptance ratio $\gamma$ defined based on the posterior distribution (7). This approach is summarized as Algorithms 2 and 3, respectively, for problems involving constant and concentration-dependent material properties, and further technical details are provided in [38].

To approximate the posterior distribution, we generate 5000 sample points in case of problem P1 and 5000 sample functions in case of problem P2 (i.e., we set $M=5000$ in Algorithms 2 and 3). The values of $C_{D}, C_{t^{+}}$and $C$ are selected based on the target values of the acceptance rate which is the ratio of the number of sample points used to construct the posterior distribution to the total number of points probed by the MCMC algorithm. In this study we set the acceptance rate as $30 \%$ for problem $\mathrm{P} 1$ and $10 \%$ for problem P2. 

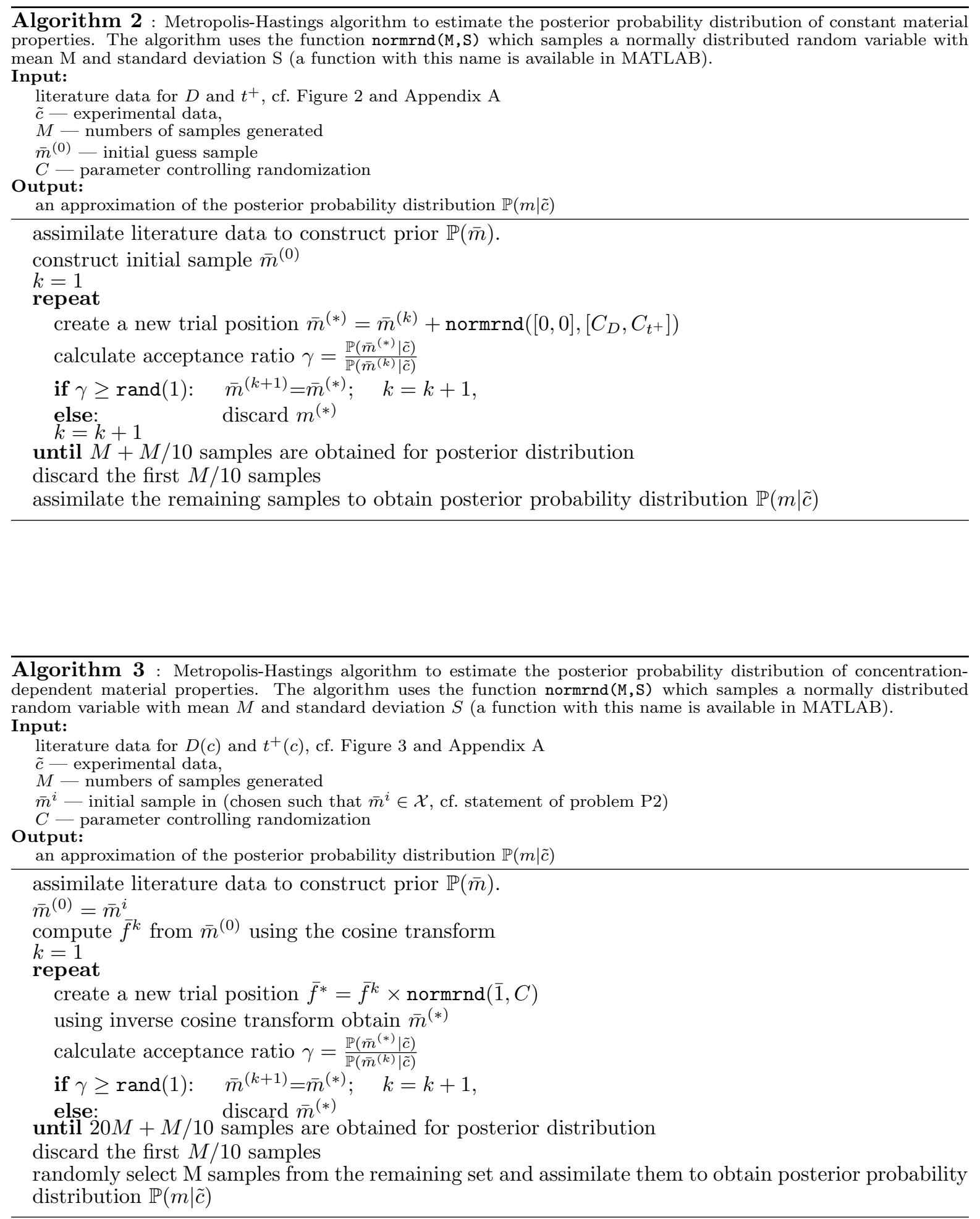


\section{References}

[1] Y. Aihara, T. Bando, H. Nakagawa, H. Yoshida, K. Hayamizu, E. Akiba, and W. S. Price. Ion transport properties of six lithium salts dissolved in $\gamma$-butyrolactone studied by self-diffusion and ionic conductivity measurements. Journal of The Electrochemical Society, 151(1):A119-A122, 2004.

[2] R. Akolkar. Mathematical model of the dendritic growth during lithium electrodeposition. Journal of Power Sources, 232:23-28, 2013.

[3] R. Akolkar. Modeling dendrite growth during lithium electrodeposition at sub-ambient temperature. Journal of Power Sources, 246:84-89, 2014.

[4] P. Bai, J. Li, F. R. Brushett, and M. Z. Bazant. Transition of lithium growth mechanisms in liquid electrolytes. Energy 85 Environmental Science, 9(10):3221-3229, 2016.

[5] JL. Barton and JO'M. Bockris. The electrolytic growth of dendrites from ionic solutions. Proc. R. Soc. Lond. A, 268(1335):485-505, 1962.

[6] C. Brissot, M. Rosso, J.-N. Chazalviel, and S. Lascaud. Dendritic growth mechanisms in lithium/polymer cells. Journal of Power Sources, 81-82:925 - 929, 1999.

[7] V. Bukshtynov and B. Protas. Optimal reconstruction of material properties in complex multiphysics phenomena. J. Comp. Phys., 242:889-914, 2013.

[8] V. Bukshtynov, O. Volkov, and B. Protas. On optimal reconstruction of constitutive relations. Physica D, 240:1228-1244, 2011.

[9] N. A. Chaturvedi, R. Klein, J. Christensenand J. Ahmed, and A. Kojic. Algorithms for advanced battery-management systems. Control Systems, IEEE, 30(3):49-68, 2010.

[10] J. N. Chazalviel. Electrochemical aspects of the generation of ramified metallic electrodeposits. Physical review A, 42(12):7355, 1990.

[11] S. Chib and E. Greenberg. Understanding the Metropolis-Hastings Algorithm. The american statistician, 49(4):327-335, 1995.

[12] T. D. Claridge. High-Resolution NMR Techniques in Organic Chemistry, volume 27. Newnes, 2008.

[13] D. A. Cogswell. Quantitative phase-field modeling of dendritic electrodeposition. Physical Review E, 92(1):011301, 2015.

[14] W. Dreyer, C. Guhlke, and R. Muller. Overcoming the shortcomings of the Nernst-Planck model. Physical Chemistry Chemical Physics, 15(19):7075-7086, 2013.

[15] D. R. Ely, A. Jana, and R E. García. Phase field kinetics of lithium electrodeposits. Journal of Power Sources, 272:581-594, 2014.

[16] W. R. Gilks, S. Richardson, and D. Spiegelhalter. Markov chain Monte Carlo in practice. CRC press, 1995.

[17] K. Hayamizu. Temperature dependence of self-diffusion coefficients of ions and solvents in ethylene carbonate, propylene carbonate, and diethyl carbonate single solutions and ethylene carbonate+ diethyl carbonate binary solutions of $\mathrm{LiPF}_{6}$ studied by NMR. Journal of Chemical 83 Engineering Data, 57(7):2012-2017, 2012.

[18] Andrew J. Ilott, Mohaddese Mohammadi, Hee Jung Chang, Clare P. Grey, and Alexej Jerschow. Real-time 3D imaging of microstructure growth in battery cells using indirect MRI. Proceedings of the National Academy of Sciences, 113(39):10779-10784, 2016. 
[19] A. Jana and R Edwin García. Lithium dendrite growth mechanisms in liquid electrolytes. Nano Energy, 41:552-565, 2017.

[20] M. Klett, M. Giesecke, A. Nyman, F. Hallberg, R. W. Lindstrom, G. Lindbergh, and I. Foro. Quantifying mass transport during polarization in a Li-ion battery electrolyte by in situ ${ }^{7} \mathrm{Li} \mathrm{NMR}$ imaging. Journal of the American Chemical Society, 134(36):14654-14657, 2012.

[21] K. Kondo, M. Sano, A. Hiwara, T. Omi, M. Fujita, A. Kuwae, M. Iida, K. Mogi, and H. Yokoyama. Conductivity and solvation of $\mathrm{Li}^{+}$ions of $\mathrm{LiPF}_{6}$ in propylene carbonate solutions. The Journal of Physical Chemistry B, 104(20):5040-5044, 2000.

[22] S. A. Krachkovskiy, A. D. Pauric, I. C. Halalay, and G. R. Goward. Slice-selective NMR diffusion measurements: a robust and reliable tool for in situ characterization of ion-transport properties in lithium-ion battery electrolytes. The Journal of Physical Chemistry Letters, 4(22):3940-3944, 2013.

[23] A. Lee, W. W. Cohen, and K. R. Koedinger. A computational model of how learner errors arise from weak prior knowledge. In Proceedings of the Annual Conference of the Cognitive Science Society, Austin, TX, pages 1288-1293, 2009.

[24] J. Liu and Ch. W. Monroe. Solute-Volume Effects in Electrolyte Transport. Electrochimica Acta, 135:447-460, 2014.

[25] L. Lu, X. Han, J. Li, J. Hua, and M. Ouyang. A review on the key issues for lithium-ion battery management in electric vehicles. Journal of Power Sources, 226:272-288, 2013.

[26] A. Mehrotra and V. Srinivasan. Transport Property measurements for $\mathrm{LiPF}_{6}$ in EC: DEC (1: 1). In Meeting Abstracts, number 14 in 1, pages 1152-1152. The Electrochemical Society, 2013.

[27] A. Mistry, C. Fear, R. Carter, C. T. Love, and P. P. Mukherjee. Electrolyte confinement alters lithium electrodepositionz. ACS Energy Letters, 4(1):156-162, 2018.

[28] C. Monroe and J. Newman. Dendrite growth in lithium/polymer systems a propagation model for liquid electrolytes under galvanostatic conditions. Journal of The Electrochemical Society, 150(10):A1377-A1384, 2003.

[29] C. W. Monroe and C. Delacourt. Continuum transport laws for locally non-neutral concentrated electrolytes. Electrochimica Acta, 114:649-657, 2013.

[30] J. Newman, K. E. Thomas, H. Hafezi, and D. R. Wheeler. Modeling of lithium-ion batteries. Journal of power sources, 119:838-843, 2003.

[31] J. Newman and K. E. Thomas-Alyea. Electrochemical Systems. John Wiley and Sons, 2004.

[32] K. Nishikawa, T. Mori, T. Nishida, Y. Fukunaka, and M. Rosso. Li dendrite growth and li+ ionic mass transfer phenomenon. Journal of electroanalytical chemistry, 661(1):84-89, 2011.

[33] J. Nocedal and S. Wright. Numerical Optimization. Springer, 2002.

[34] A. Nyman, M. Behm, and G. Lindbergh. Electrochemical Characterisation and Modelling of the Mass Transport Phenomena in $\mathrm{LiPF}_{6}-E C-E M C$ Electrolyte. Electrochimica Acta, 53(22):63566365,2008 .

[35] M. Park, X. Zhang, M. Chung, G. B. Less, and A. M. Sastry. A review of conduction phenomena in li-ion batteries. Journal of Power Sources, 195(24):7904-7929, 2010. 
[36] G. Richardson, J. M. Foster, A. K. Sethurajan, S. A. Krachkovskiy, I. C. Halalay, G. R. Goward, and B. Protas. The effect of ionic aggregates on the transport of charged species in lithium electrolyte solutions. Journal of The Electrochemical Society, 165(9):H561-H567, 2018.

[37] M. Riley, P. S. Fedkiw, and S. A. Khan. Transport properties of lithium hectorite-based composite electrolytes. Journal of the Electrochemical Society, 149(6):A667-A674, 2002.

[38] A. Sethurajan, S. Krachkovskiy, G. Goward, and B. Protas. Bayesian Uncertainty Quantification in Inverse Modelling of Electrochemical Systems. Journal of Computational Chemistry, 2018. (in press).

[39] A. K. Sethurajan. Reconstruction of concentration-dependent material properties in electrochemical systems. Master's thesis, McMaster University, 2014.

[40] A. K. Sethurajan, S. A. Krachkovskiy, I. C. Halalay, G. R. Goward, and B. Protas. Accurate characterization of ion transport properties in binary symmetric electrolytes using in situ $\mathrm{nmr}$ imaging and inverse modeling. The Journal of Physical Chemistry B, 119(37):12238-12248, 2015.

[41] R.C. Smith. Uncertainty Quantification: Theory, Implementation, and Applications. Computational Science and Engineering. SIAM, 2013.

[42] J. Steiger. Mechanisms of dendrite growth in lithium metal batteries. PhD thesis, Karlsruher Institut fur Technologie, 2015.

[43] J. Steiger, D. Kramer, and R. Mönig. Microscopic observations of the formation, growth and shrinkage of lithium moss during electrodeposition and dissolution. Electrochimica Acta, 136:529536,2014 .

[44] S. Stewart and J. Newman. Measuring the salt activity coefficient in lithium-battery electrolytes. Journal of The Electrochemical Society, 155(6):A458-A463, 2008.

[45] A. M. Stuart. Inverse problems: A Bayesian perspective. Acta Numerica, 19:451-559, 2010.

[46] L. Tenorio. An Introduction to Data Analysis and Uncertainty Quantification for Inverse Problems. Society for Industrial and Applied Mathematics, Philadelphia, PA, 2017.

[47] L. O. Valoen and J. N. Reimers. Transport properties of $\mathrm{LiPF}_{6}$-based Li-ion battery electrolytes. Journal of The Electrochemical Society, 152(5):A882-A891, 2005.

[48] X. Wang, W. Zeng, L. Hong, W. Xu, H. Yang, F. Wang, H. Duan, M. Tang, and H. Jiang. Stress-driven lithium dendrite growth mechanism and dendrite mitigation by electroplating on soft substrates. Nature Energy, 3(3):227-235, 2018.

[49] K. N. Wood, E. Kazyak, A. F. Chadwick, K. Chen, J. Zhang, K. Thornton, and N. P. Dasgupta. Dendrites and pits: Untangling the complex behavior of lithium metal anodes through operando video microscopy. ACS Central Science, 2(11):790-801, 2016.

[50] J. Zhang, W. Xu, and W. A. Henderson. Lithium metal anodes and rechargeable lithium metal batteries. Springer International Publishing Switzerland, 2017.

[51] S. S. Zhang. A review on electrolyte additives for lithium-ion batteries. Journal of Power Sources, 162(2):1379-1394, 2006.

[52] J. Zhao, L. Wang, X. He, C. Wan, and C. Jiang. Determination of lithium-ion transference numbers in $\mathrm{LiPF}_{6}-\mathrm{PC}$ solutions based on electrochemical polarization and NMR measurements. Journal of The Electrochemical Society, 155(4):A292-A296, 2008. 
[53] S. Zugmann, M. Fleischmann, M. Amereller, R. M. Gschwind, M. Winter, and H. J. Gores. Salt diffusion coefficients, concentration dependence of cell potentials, and transference numbers of lithium difluoromono (oxalato) borate-based solutions. Journal of Chemical \& Engineering Data, 56(12):4786-4789, 2011. 


\begin{tabular}{|c|c|c|c|}
\hline Planck-Nernst Model & Sink Function $\mathcal{F}_{l(t)}(x)$ & Inverse Problem & $\mathcal{J}\left(\left[\widehat{D}, \widehat{t}^{+}\right]\right)$ \\
\hline standard, Eq. (1) & - & P1 & 2.61 \\
\hline modified, Eq. (2) & case A, Eq. (3) & P1 & 2.26 \\
\hline modified, Eq. (2) & case B, Eq. (4) & P1 & 2.12 \\
\hline modified, Eq. (2) & case C, Eq. (5) & P1 & 2.21 \\
\hline modified, Eq. (2) & case C, Eq. (5) & P2 & 1.14 \\
\hline
\end{tabular}

Table 1: Final values of the error functional $\mathcal{J}\left(\left[\widehat{D}, \widehat{t}^{+}\right]\right)$obtained in the different cases considered. 


\section{List of Figures}

1 Schematic illustration of the electrochemical cell (a) at the beginning of the experiment (at time $t=0$ ) when there is no dendritic growth and (b) in the course of the experiment (i.e., for $t>0$ ) when the dendritic region grows from the negative (right) electrode. The standard Planck-Nernst model (1) is defined on the domain illustrated in panel (a), whereas the modification of this model given in (2) accounts for the presence of the dendritic region shown in panel $(b) . \ldots \ldots \ldots \ldots$

2 Values of the constant diffusion coefficient $D$ and the transference number $t^{+}$reported in the literature for $\mathrm{LiPF}_{6}-\mathrm{EC} / \mathrm{DMC}[17,20,26,30,44,47,53]$. When the values of both $D$ and $t^{+}$are reported, they are represented with a symbol, whereas if the value of $D$ or $t^{+}$is reported only, it is represented with a vertical or a horizontal line. . . . . . . . .

3 (a) Dependence of the diffusion coefficient $D(c)$ on the concentration $c$ reported in the literature for $\mathrm{LiPF}_{6}-\mathrm{EC} / \mathrm{DMC}[1,21,22,26,52]$. (b) Dependence of the transference number $t^{+}(c)$ on the concentration $c$ reported in the literature for $\mathrm{LiPF}_{6}-\mathrm{EC} / \mathrm{DMC}$

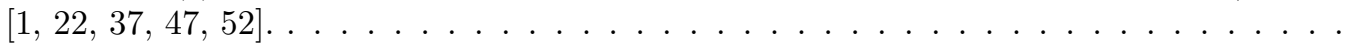

4 Concentration profiles obtained via in-situ magnetic resonance imaging during a galvanostatic polarization experiment. The shrinking of the region where the concentration profiles are acquired with time is evident near the negative (right) electrode. . . . . . . .

5 Dependence of the location $l(t)$ of the left boundary of the dendritic region, cf. figure 1(b), on time $t$ for different applied currents $I \ldots \ldots \ldots \ldots \ldots$

6 The "weak" prior $\mathbb{P}\left(\left[D, t^{+}\right]\right)$constructed as described in Appendix $\mathrm{C}$ based on the literature data for diffusivity and the transference numbers, cf. Figures 2 and 3. Since in the calculation of the posterior suitable normalization is ensured by the expression in the denominator in (7), for simplicity, the prior shown here has an arbitrary scaling. . . . .

7 (red solid lines) Reconstructions of constant material properties $\left[\widehat{D}, \widehat{t}^{+}\right]$using (a) the standard Planck-Nernst model (1), (b) modified model (2) with sink function (3) (case A), (c) modified model (2) with sink function (4) (case B), and (d) modified model (2) with sink function (5) (case C). The associated posterior probability distributions are indicted with filled contours. The black contour lines indicate the boundaries of the credibility regions with the indicated credibility values. Information about the values of the material properties reported in the literature, cf. Figure 2, is contained in the posterior probability distribution through the choice of the prior, cf. relation (7) and Figure 6. . . . . . . . . . . . . . . . . . . .

8 (a) Sink functions with different slopes $s=\frac{d}{d x} \mathcal{F}_{l(t)}(x), x \in[l(t), L]$, varying continuously from $-\infty$ to $+\infty$ (the vertical arrows indicate the trends with the increase of $s$ whereas the thick red lines represent the standard Planck-Nernst model (1) and the different variants of the modified model (2) arising as special cases for particular values of $s$ ); (b) transference numbers $\widehat{t}^{+}$inferred by solving problem $\mathrm{P} 1$ as function of the slope $s$ characterizing the sink function $\mathcal{F}_{l(t)}(x)$, cf. panel (a). . . . . . . . . . . . . .

$9 \quad$ Experimental concentration profiles $\tilde{c}\left(x, t_{i}\right)$ (symbols) and the concentration profiles predicted by (left column, solid lines) the standard Planck-Nernst model (1) using the optimal constant material properties $\left[\widehat{D}, \widehat{t}^{+}\right]=\left[3.60 \times 10^{-10},-0.211\right]$, (middle column, solid lines) the modified Planck-Nernst model (2) with a uniform sink function (5) and using the optimal constant material properties $\left[\widehat{D}, \widehat{t}^{+}\right]=\left[1.84 \times 10^{-10}, 0.203\right]$, and (right column, solid lines) the modified Planck-Nernst model (2) with a uniform sink function (5) and using the optimal concentration-dependent material properties $\left[\widehat{D}(c), \widehat{t}^{+}(c)\right]$ from

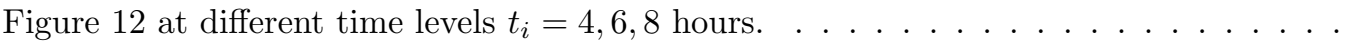

10 The datasets are the same as in Figure 9, here shown at the time levels $t_{i}=10,12,14$

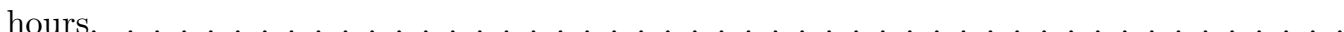


11 The volume fractions $1-\phi(x, t)$ occupied by lithium dendrites predicted by the modified Planck-Nernst model (2) with a uniform sink function (5) as functions of $x \in[l(T), L]$ for different times spread uniformly between 0 and $T$ (the arrow indicates the trend with the increase of time $t) \ldots \ldots \ldots \ldots \ldots$

12 Reconstruction of concentration-dependent (a) diffusivity $\widehat{D}(c)$ and (b) transference number $\widehat{t}^{+}(c)$ (black solid lines) together with the associated posterior probability distributions (filled contours) as functions of the concentration $c$ for the modified Planck-Nernst model (2) with the uniform sink function (5), case C. The black contour lines indicate the boundaries of the credibility regions with the indicated credibility values. Information about the values of the material properties reported in the literature, cf. Figure 3 , is contained in the posterior probability distribution through the choice of the prior, cf. relation $(7)$ and Figure $6 \ldots \ldots \ldots \ldots \ldots$ 


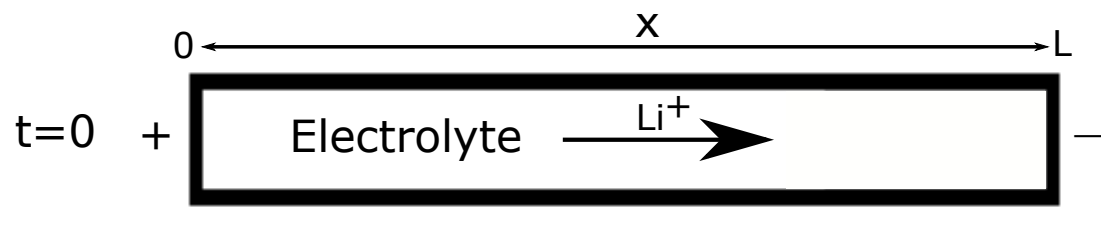

(a)

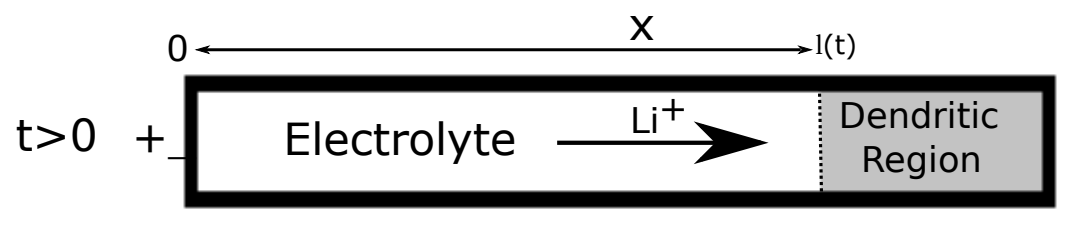

(b)

Figure 1: Schematic illustration of the electrochemical cell (a) at the beginning of the experiment (at time $t=0$ ) when there is no dendritic growth and (b) in the course of the experiment (i.e., for $t>0$ ) when the dendritic region grows from the negative (right) electrode. The standard Planck-Nernst model (1) is defined on the domain illustrated in panel (a), whereas the modification of this model given in (2) accounts for the presence of the dendritic region shown in panel (b). 


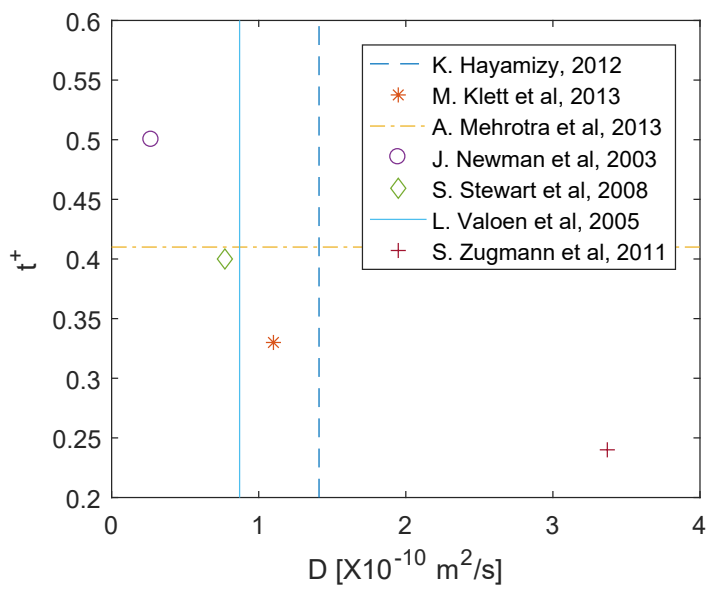

Figure 2: Values of the constant diffusion coefficient $D$ and the transference number $t^{+}$reported in the literature for $\mathrm{LiPF}_{6}-\mathrm{EC} / \mathrm{DMC}[17,20,26,30,44,47,53]$. When the values of both $D$ and $t^{+}$are reported, they are represented with a symbol, whereas if the value of $D$ or $t^{+}$is reported only, it is represented with a vertical or a horizontal line. 


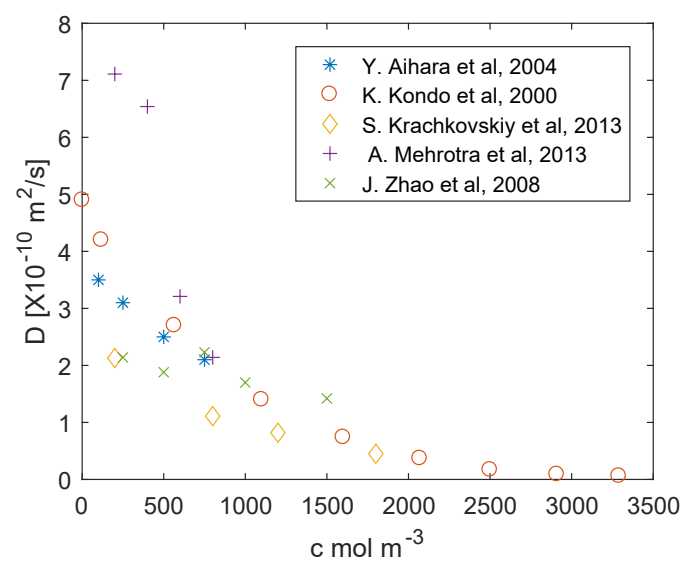

(a)

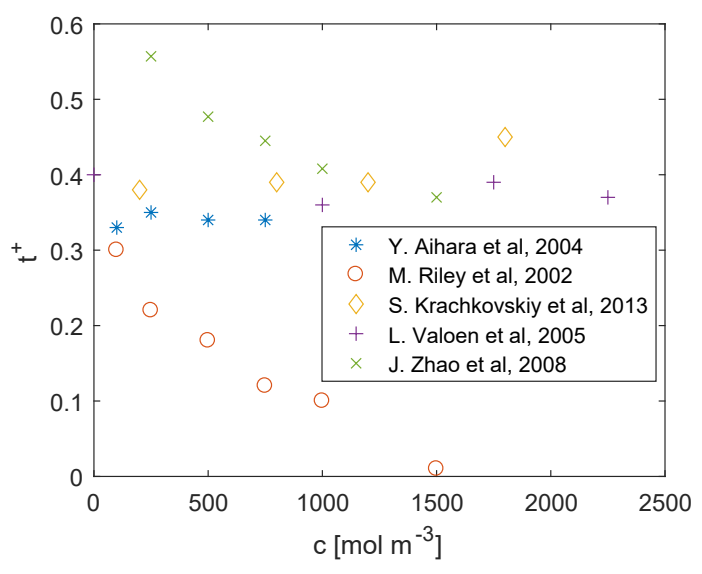

(b)

Figure 3: (a) Dependence of the diffusion coefficient $D(c)$ on the concentration $c$ reported in the literature for $\mathrm{LiPF}_{6}-\mathrm{EC} / \mathrm{DMC}[1,21,22,26,52]$. (b) Dependence of the transference number $t^{+}(c)$ on the concentration $c$ reported in the literature for $\mathrm{LiPF}_{6}-\mathrm{EC} / \mathrm{DMC}[1,22,37,47,52]$. 


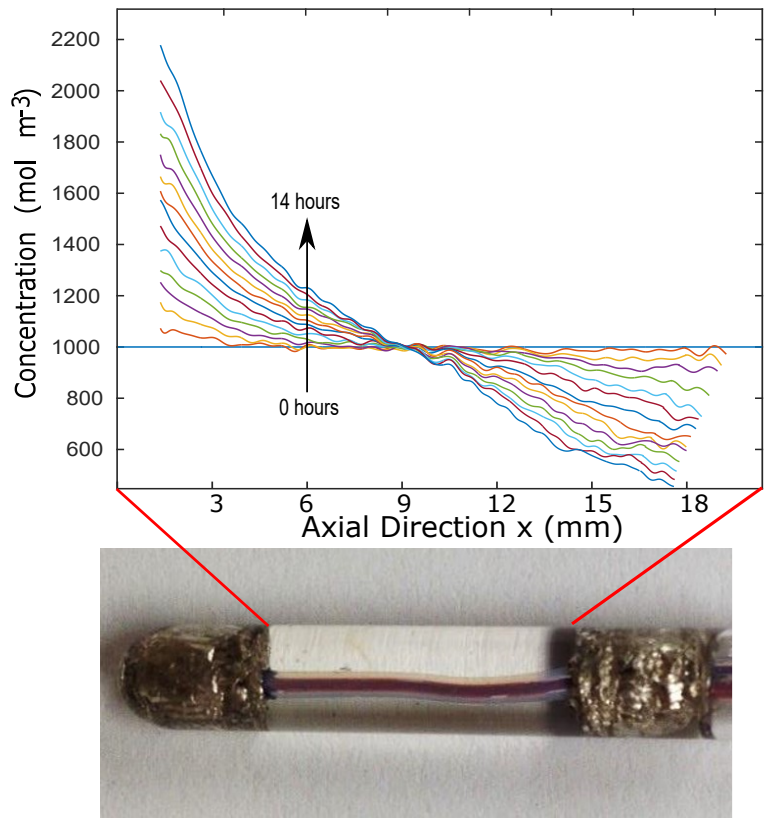

Figure 4: Concentration profiles obtained via in-situ magnetic resonance imaging during a galvanostatic polarization experiment. The shrinking of the region where the concentration profiles are acquired with time is evident near the negative (right) electrode. 


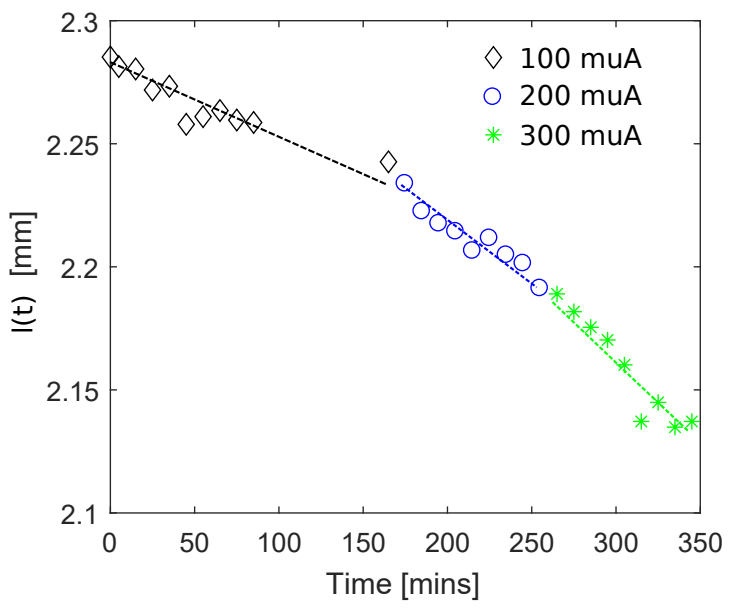

Figure 5: Dependence of the location $l(t)$ of the left boundary of the dendritic region, cf. figure 1(b), on time $t$ for different applied currents $I$. 

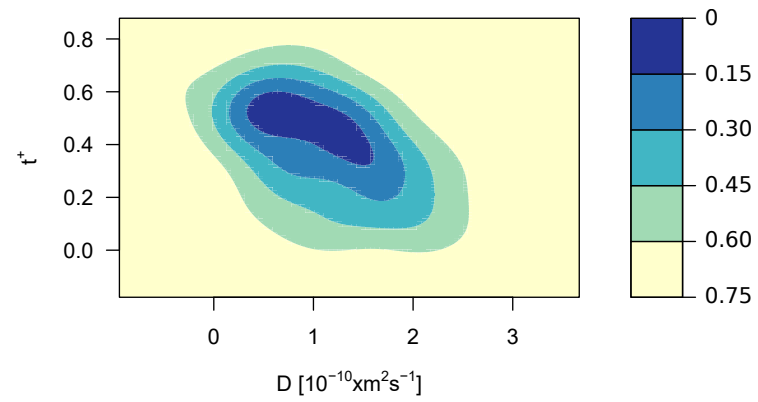

Figure 6: The "weak" prior $\mathbb{P}\left(\left[D, t^{+}\right]\right)$constructed as described in Appendix $\mathrm{C}$ based on the literature data for diffusivity and the transference numbers, cf. Figures 2 and 3. Since in the calculation of the posterior suitable normalization is ensured by the expression in the denominator in (7), for simplicity, the prior shown here has an arbitrary scaling. 


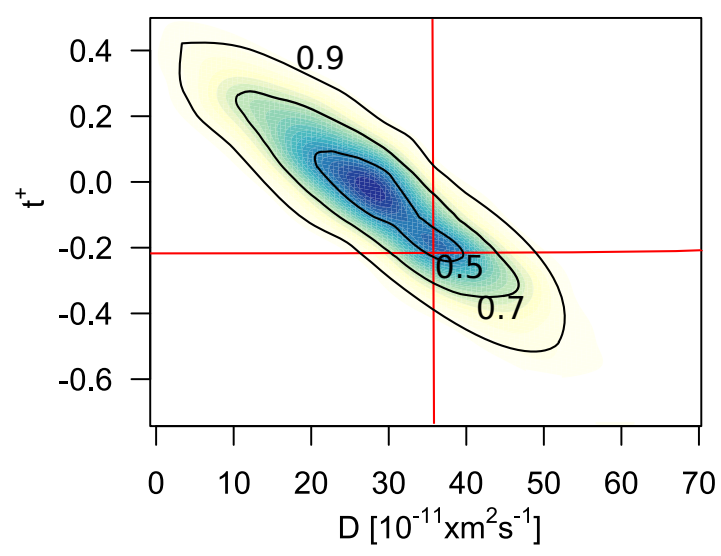

(a)

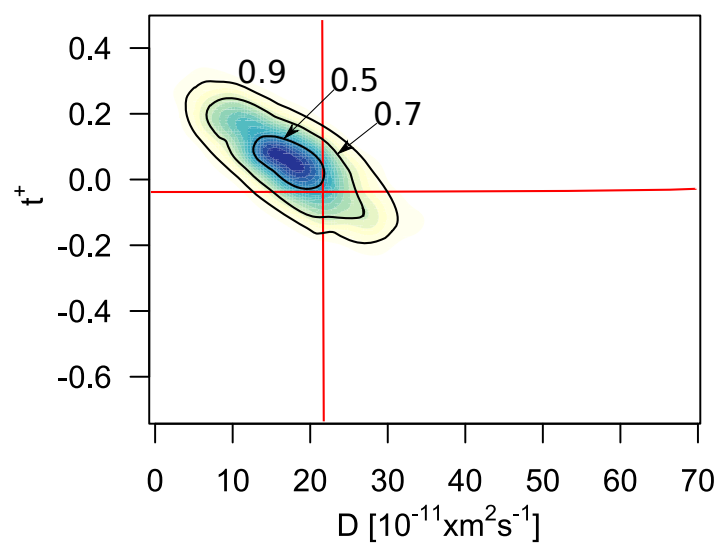

(c)

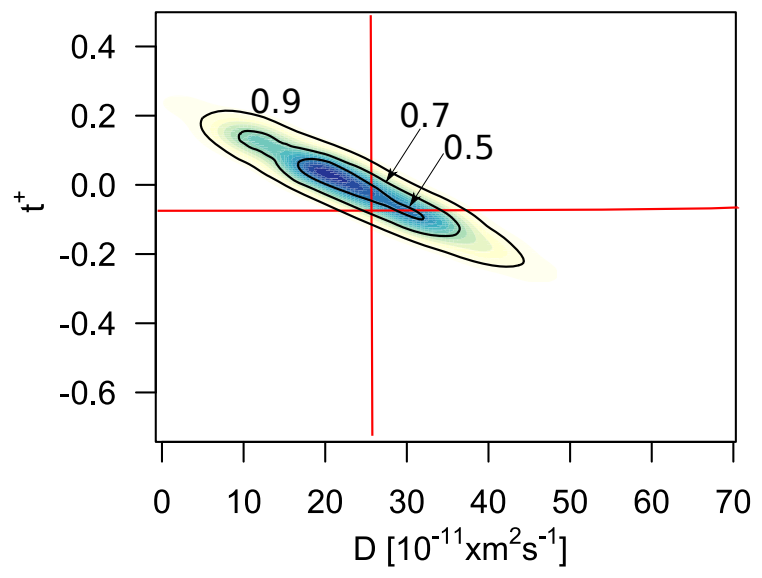

(b)

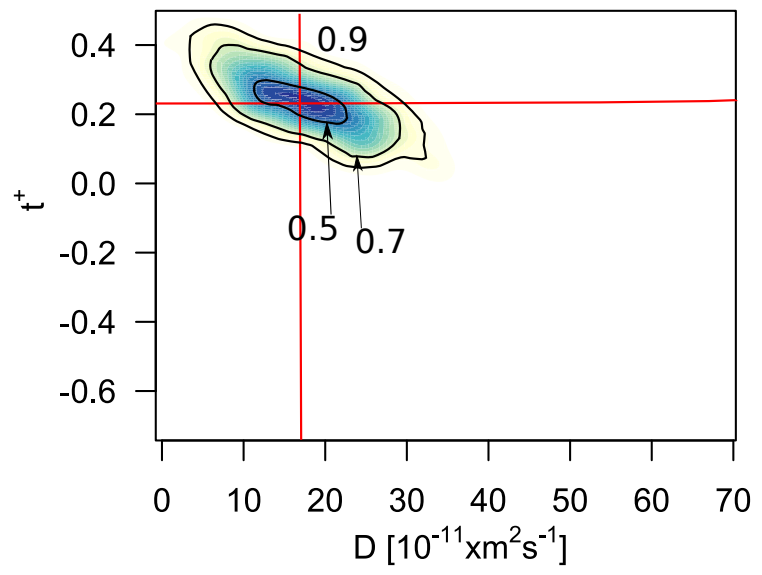

(d)

Figure 7: (red solid lines) Reconstructions of constant material properties $\left[\widehat{D}, \widehat{t}^{+}\right]$using (a) the standard Planck-Nernst model (1), (b) modified model (2) with sink function (3) (case A), (c) modified model (2) with sink function (4) (case B), and (d) modified model (2) with sink function (5) (case C). The associated posterior probability distributions are indicted with filled contours. The black contour lines indicate the boundaries of the credibility regions with the indicated credibility values. Information about the values of the material properties reported in the literature, cf. Figure 2, is contained in the posterior probability distribution through the choice of the prior, cf. relation (7) and Figure 6. 


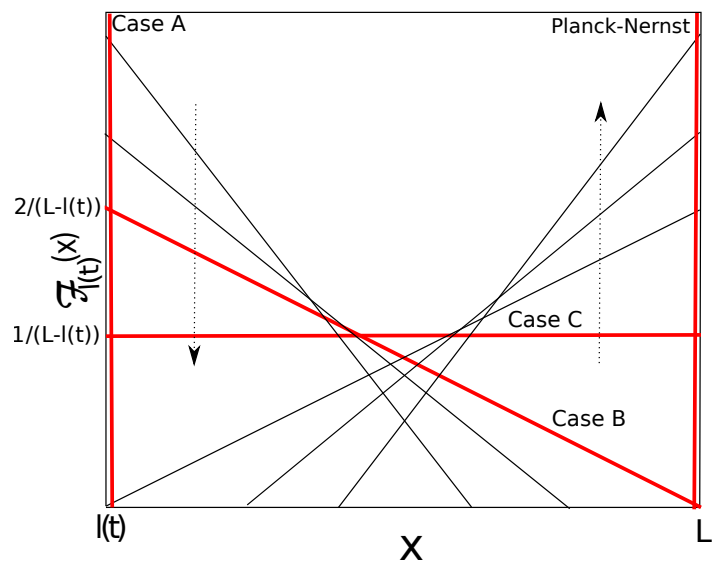

(a)

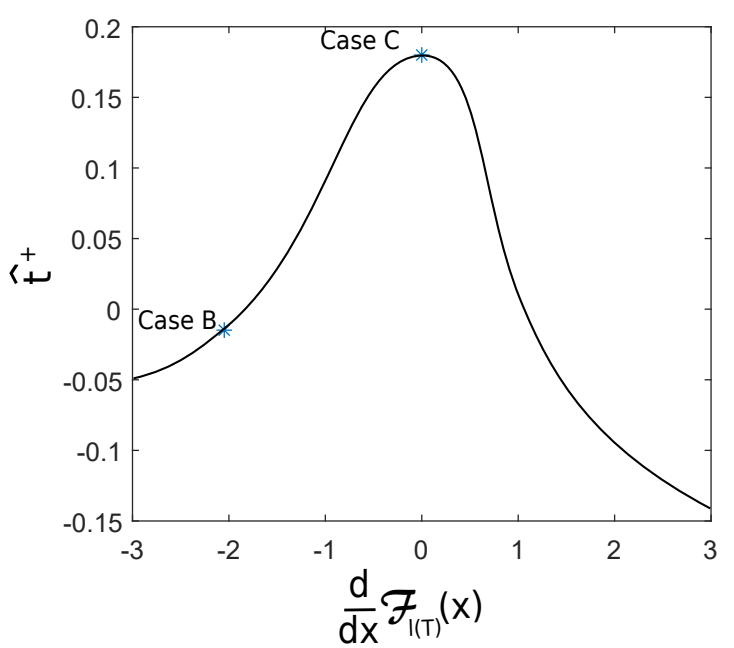

(b)

Figure 8: (a) Sink functions with different slopes $s=\frac{d}{d x} \mathcal{F}_{l(t)}(x), x \in[l(t), L]$, varying continuously from $-\infty$ to $+\infty$ (the vertical arrows indicate the trends with the increase of $s$ whereas the thick red lines represent the standard Planck-Nernst model (1) and the different variants of the modified model (2) arising as special cases for particular values of $s$ ); (b) transference numbers $\hat{t}^{+}$inferred by solving problem P1 as function of the slope $s$ characterizing the sink function $\mathcal{F}_{l(t)}(x)$, cf. panel (a). 

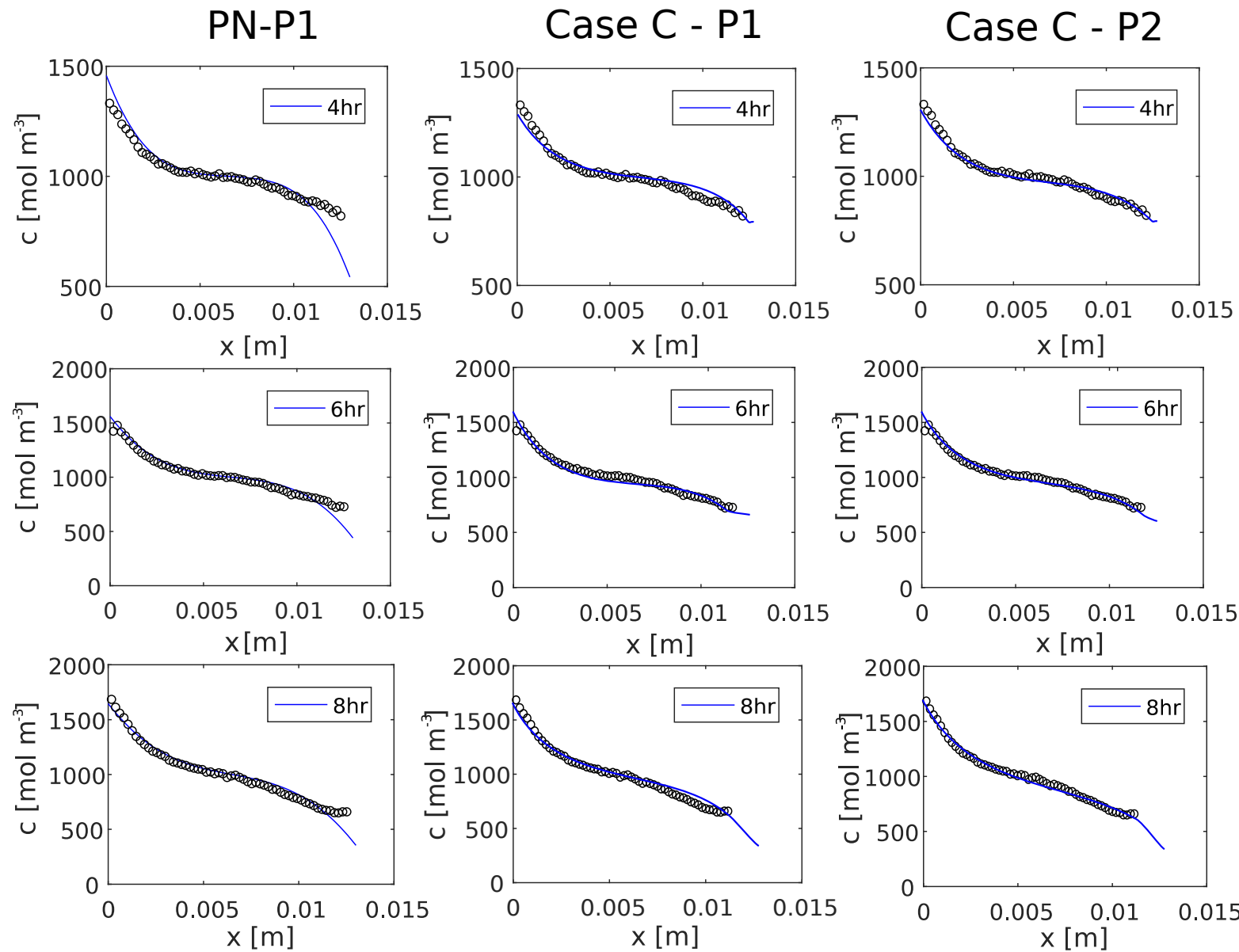

Figure 9: Experimental concentration profiles $\tilde{c}\left(x, t_{i}\right)$ (symbols) and the concentration profiles predicted by (left column, solid lines) the standard Planck-Nernst model (1) using the optimal constant material properties $\left[\widehat{D}, \widehat{t}^{+}\right]=\left[3.60 \times 10^{-10},-0.211\right]$, (middle column, solid lines) the modified Planck-Nernst model (2) with a uniform sink function (5) and using the optimal constant material properties $\left[\widehat{D}, \widehat{t}^{+}\right]=$ $\left[1.84 \times 10^{-10}, 0.203\right]$, and (right column, solid lines) the modified Planck-Nernst model (2) with a uniform sink function (5) and using the optimal concentration-dependent material properties $\left[\widehat{D}(c), \widehat{t}^{+}(c)\right]$ from Figure 12 at different time levels $t_{i}=4,6,8$ hours. 

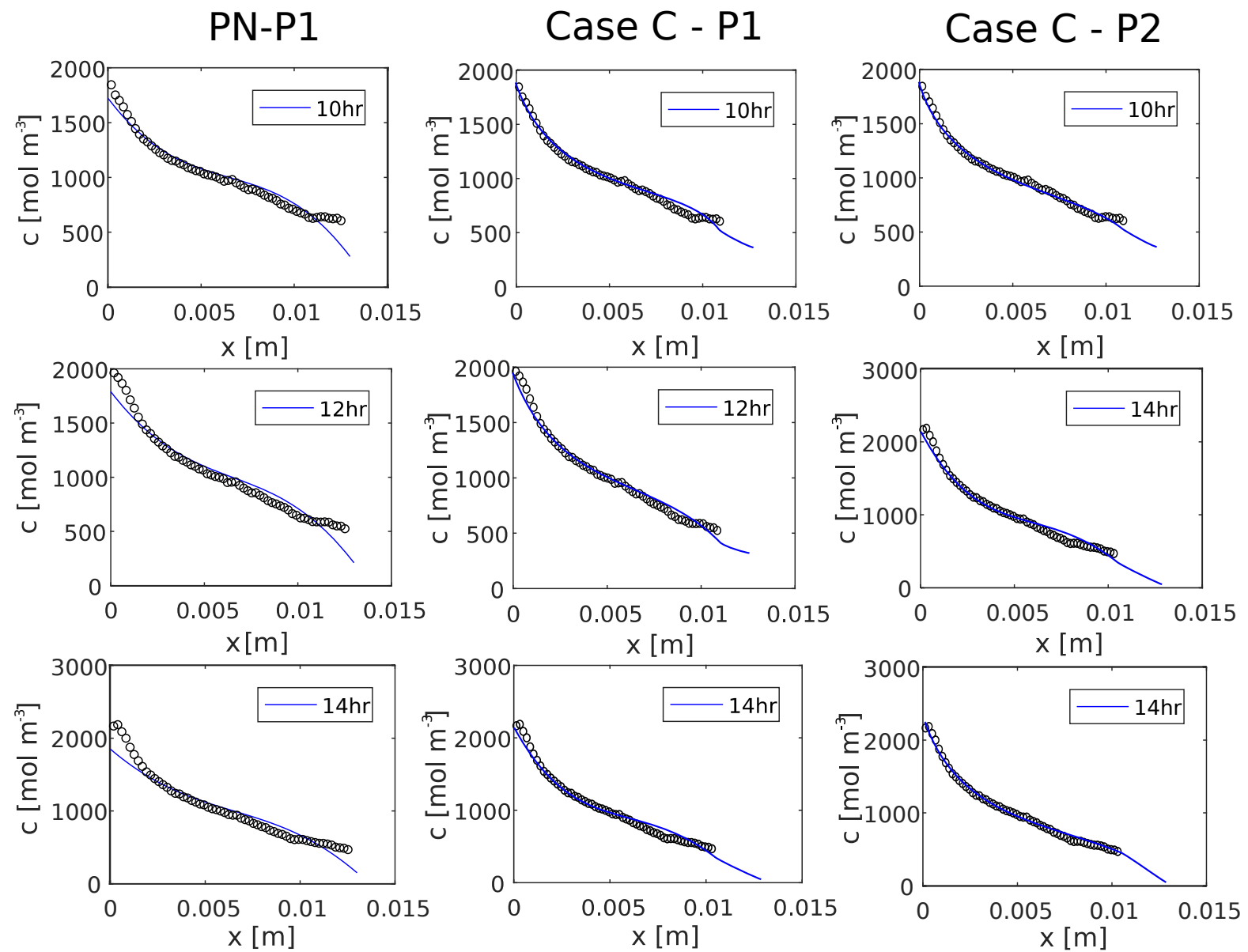

Figure 10: The datasets are the same as in Figure 9, here shown at the time levels $t_{i}=10,12,14$ hours. 


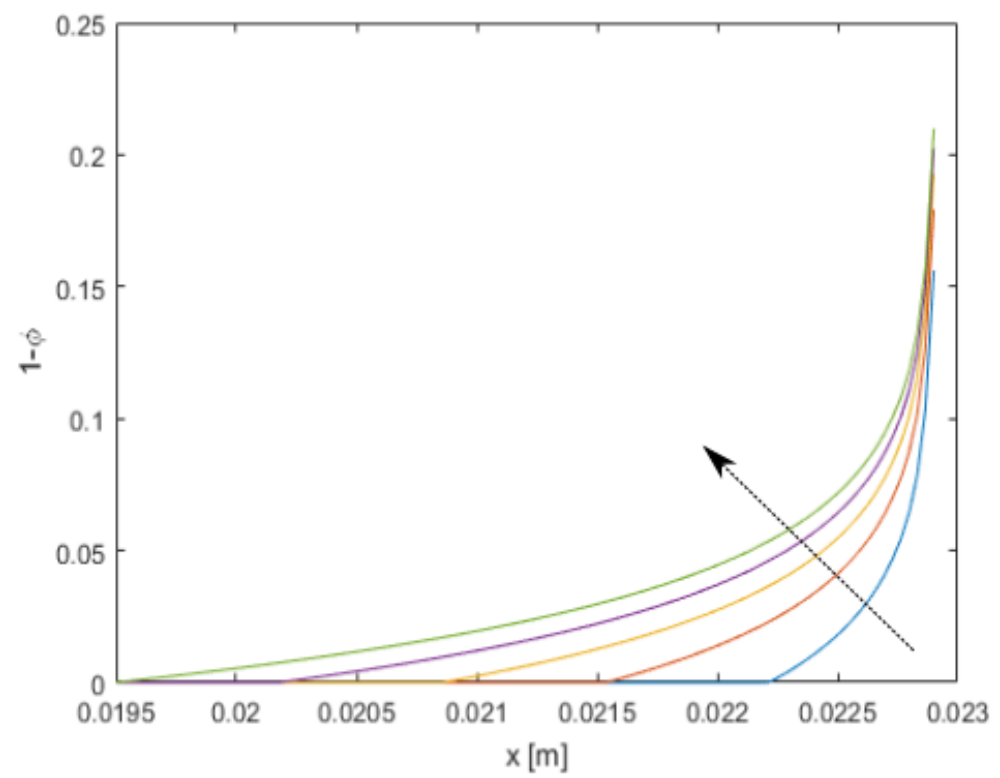

Figure 11: The volume fractions $1-\phi(x, t)$ occupied by lithium dendrites predicted by the modified Planck-Nernst model (2) with a uniform sink function (5) as functions of $x \in[l(T), L]$ for different times spread uniformly between 0 and $T$ (the arrow indicates the trend with the increase of time $t$ ). 


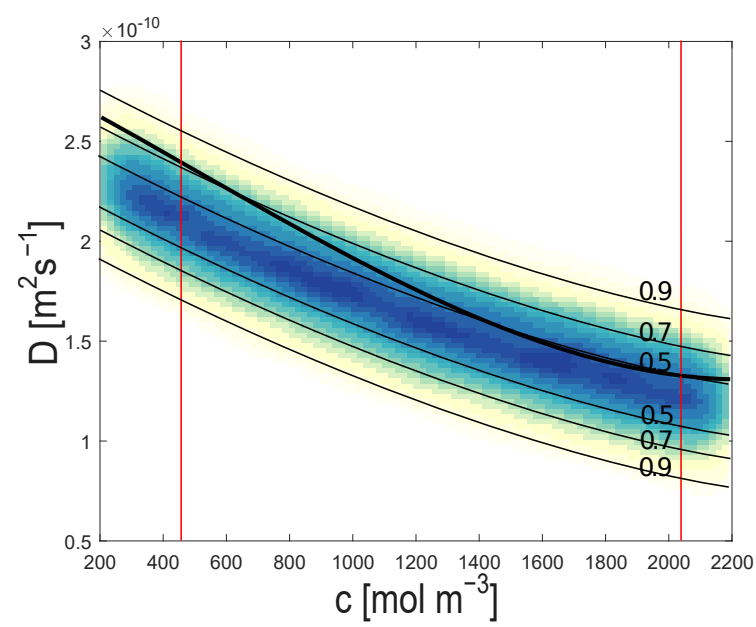

(a)

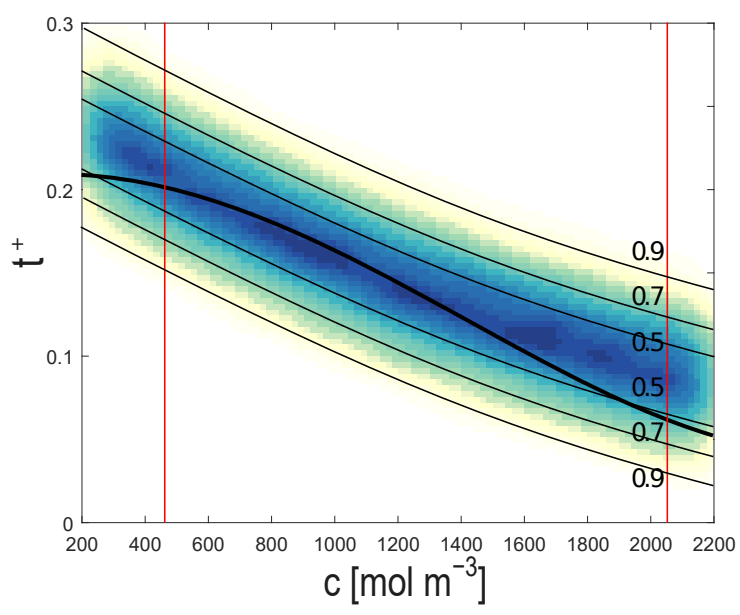

(b)

Figure 12: Reconstruction of concentration-dependent (a) diffusivity $\widehat{D}(c)$ and (b) transference number $\widehat{t}^{+}(c)$ (black solid lines) together with the associated posterior probability distributions (filled contours) as functions of the concentration $c$ for the modified Planck-Nernst model (2) with the uniform sink function (5), case C. The black contour lines indicate the boundaries of the credibility regions with the indicated credibility values. Information about the values of the material properties reported in the literature, cf. Figure 3, is contained in the posterior probability distribution through the choice of the prior, cf. relation (7) and Figure 6. 